3 Wei-Bin Shen ${ }^{1 \#}$, James Logue ${ }^{2 \#}$, Penghua Yang ${ }^{1 \#}$, Lauren Baracco ${ }^{2}$, Montasir Elahi $^{1}$, E.

\section{SARS-CoV-2 invades cognitive centers of the brain and induces Alzheimer's-like}

\section{neuropathology}

4

6

7

Albert Reece ${ }^{1,3}$, Bingbing Wang ${ }^{1}$, Ling $\mathrm{Li}^{4}$, Thomas G Blanchard ${ }^{4}$, Zhe Han ${ }^{5}$, Matthew B

Frieman $^{2}$, Robert A Rissman ${ }^{6,7}$, Peixin Yang ${ }^{1,3 *}$

${ }^{1}$ Department of Obstetrics, Gynecology \& Reproductive Sciences, University of Maryland School of Medicine, Baltimore, Maryland, USA.

${ }^{2}$ Department of Microbiology and Immunology, University of Maryland School of Medicine, Baltimore, MD 21201, USA.

${ }^{3}$ Department of Biochemistry \& Molecular Biology, University of Maryland School of Medicine, Baltimore, Maryland, USA.

${ }^{4}$ Department of Pediatrics, University of Maryland School of Medicine, Baltimore, MD, USA.

${ }^{5}$ Department of Medicine, University of Maryland School of Medicine, Baltimore, MD, USA.

${ }^{6}$ Department of Neurosciences, University of California San Diego, La Jolla, CA, 92093.

${ }^{7}$ Shiley-Marcos Alzheimer's Disease Research Center, University of California San Diego, La Jolla, California, 92093.

\# These authors contribute equally to this work.

*Correspondence: Peixin Yang, PhD, Department of Obstetrics, Gynecology \& Reproductive Sciences, University of Maryland School of Medicine, BRB11-039, 655 W. Baltimore Street, Baltimore, MD, 21201. Email:

pyang@som.umaryland.edu, Tel: 410-706-8402, Fax: 410-706-5747.

Conflict of interest statement: The authors have declared that no conflict of interest exists Subheading: Neurodegenerative phenotypes in COVID-19

Keywords: SARS-CoV-2; neurons; iPSC; Alzheimer's; COVID-19 brains; Alzheimer's infectious etiology; betaamyloid; neuroinflammation; tauopathy. 


\section{$\underline{\text { Abstract }}$}

Major cell entry factors of SARS-CoV-2 are present in neurons; however, the neurotropism of

SARS-CoV-2 and the phenotypes of infected neurons are still unclear. Acute neurological disorders occur in many patients, and one-third of COVID-19 survivors suffer from "brain diseases". Here, we show that

SARS-CoV-2 invades the brains of five patients with COVID-19 and Alzheimer's, autism, frontotemporal dementia or no underlying condition by infecting neurons and other cells in the cortex. SARS-CoV-2 induces or enhances Alzheimer's-like neuropathology with manifestations of $\beta$-amyloid aggregation and plaque formation, tauopathy, neuroinflammation and cell death. SARS-CoV-2 infects mature but not immature neurons derived from inducible pluripotent stem cells from healthy and Alzheimer's individuals through its receptor ACE2 and facilitator neuropilin-1. SARS-CoV-2 triggers Alzheimer's-like gene programs in healthy neurons and exacerbates Alzheimer's neuropathology. A gene signature defined as an Alzheimer's infectious etiology is identified through SARS-CoV-2 infection, and silencing the top three downregulated genes in human primary neurons recapitulates the neurodegenerative phenotypes of SARSCoV-2. Thus, SARS-CoV-2 invades the brain and activates an Alzheimer's-like program. 


\section{Introduction}

The COVID-19 pandemic caused by severe acute respiratory syndrome coronavirus 2 (SARS-CoV2) has infected at least 213 million people worldwide and 37.8 million Americans to date. SARS-CoV-2 not only causes respiratory syndromes but also leads to neurological abnormalities, with an $85 \%$ occurrence rate in patients with Alzheimer's disease [1, 2]. In fact, neurological symptoms, including hypogeusia, headache and anosmia, precede the onset of respiratory symptoms in the majority of patients with COVID-19. The major chronic sequelae of COVID-19 are expected to be cognitive decline and dementia [3]. A recent study has shown that one-third of COVID-19 survivors exhibit substantial neurological and psychiatric morbidity in the 6 months after SARS-CoV-2 infection [4]. Furthermore, the "brain disease" risk is not limited to patients who have severe COVID-19 [4]. Thus, the COVID-19 pandemic provides a unique but unwelcomed opportunity to study the contribution of SARS-CoV-2 to neurological disorders, including Alzheimer's disease.

It has been proposed that $\beta$-coronaviruses, including SARS-CoV-2, can invade the central nervous system [5]. The two coronaviruses closely related to SARS-CoV-2, Middle Eastern respiratory syndrome coronavirus (MERS-CoV) and severe acute respiratory syndrome coronavirus 1 (SARS-CoV-1), can infect the central nervous system [6,7]. A recent study shows the presence of SARS-CoV-2 in a patient's olfactory mucosa and its neuronal projections [8]. SARS-CoV-2 RNA is present in $36.4 \%$ of brain biopsies of fatal COVID-19 cases [9]. A recent study observes the presence of SARS-CoV-2 spike protein in some brain regions in all three COVID-19 cases studied [10]. In transgenic hACE2 mice, SARS-CoV-2 is massively present in the brain at post infection day 5 [11]. In addition to olfactory transmucosal entry of SARS-CoV-2 into the central nervous system (CNS) [8], other potential routes of SARS-CoV-2 brain entry, including blood-brain barrier (BBB) passage, especially under conditions of a compromised BBB, such as in cases of Alzheimer's disease and autism [12,13], and the infiltration of infected immune cells 
have been proposed [14], though evidence for infected immune cells remains scarce. However, neurological disorders are not limited to severe COVID-19 cases, suggesting that multiple brain entries may account for SARS-CoV-2 neurotropism. Although SARS-CoV-2 can enter the brain, experimental evidence of its presence in key brain regions involved in cognitive functions is still lacking, and little is known about the functional impact of SARS-CoV-2 CNS invasion on neurons.

SARS-CoV-2 entry into host cells is facilitated by its cell surface receptors. The spike proteins of both SARS-CoV-2 and the earlier SARS-CoV-1 bind to angiotensin-converting enzyme 2 (ACE2) as the first step of cellular entry [15]. The presence of ACE2 in neurons of the brain has been demonstrated [16]. However, ACE2 is expressed at relatively low levels in most tissues [17]. SARS-CoV-2 exhibits much higher infectivity than SARS-CoV-1. This evidence implicates either the existence of other cell surface receptors in SARS-CoV-2 cell entry or the facilitation of cell entry by other factors. Indeed, neuropilin-1 (NRP1) has been identified as a facilitator of SARS-CoV-2 entry when ACE2 is expressed, leading to high rates of infection [17]. NRP1 effectively binds to the protease furin-cleaved spike protein of SARS-CoV2 [17], a process that does not occur for SARS-CoV-1. NRP1 is abundantly expressed in cells of many tissues including neurons [18]. The presence of ACE2 and NRP1 in neurons suggests the high possibility of neurotropism. It is of interest to determine in which cell types in the CNS and at which stage of neural development SARS-CoV-2 can exert its infectibility.

There is a profound link between SARS-CoV-2 infection and dementia/Alzheimer's disease. The 2002 and 2012 SARS and MERS epidemics caused memory impairment in many recovered patients [19]. Neurological syndromes related to Alzheimer's disease, including neuroinflammatory syndromes [20, 21], seizures [22], delirium [23, 24], and alterations in personality, behavior, and cognitive deficits [20, 25], frequently occur in patients with COVID-19 who recover from COVID-19. Thus, SARS-CoV-2 infection of the brain may increase the risk of Alzheimer's disease. The direct effects of SARS-CoV-2 on neuronal 
93

94 function and survival, the inflammatory cytokine response, and hypoxia may lead to an Alzheimer's-like manifestation [26, 27]. Additionally, Alzheimer's patients have twice the risk of contracting COVID-19, which deteriorates Alzheimer's symptoms and increases mortality [28]. The cellular mechanism underlying the possible neurotropism of SARS-CoV-2 needs to be revealed for the development of possible treatments for a large number of COVID-19 survivors suffering from neurological disorders.

In this study, we sought to test whether SARS-CoV-2 is neurotropic and infects neural cells in the cognitive center in five patients with Alzheimer's disease, autism, frontotemporal dementia and no underlying condition, respectively. SARS-CoV-2 infection alters transcriptomic landscapes in favor of the development of Alzheimer's-like neuropathology in non-Alzheimer's individuals and exacerbates Alzheimer's neuropathology in patients with Alzheimer's. We found that SARS-CoV-2 invades the cognitive centers of all five COVID-19 patients, leading to Alzheimer's-like neuropathology or Alzheimer's neuropathology exacerbation. SARS-CoV-2 infects human inducible pluripotent stem cell (iPSC)-derived mature neurons from healthy individuals, leading to amyloid beta (A $\beta$ ) deposition, increased inflammation, neuronal death and increased expression of Alzheimer's mediators. Strikingly, we found that SARS-CoV-2-infects neurons from healthy individuals through a shared gene expression program with Alzheimer's neurons, leading to activation of the infectious pathways and supporting the infectious etiology of Alzheimer's disease. 


\section{Results}

112

113

114

115

116

117

\section{SARS-CoV-2 invades cognitive centers of the brain}

Because ACE2, the SARS-CoV-2 cellular receptor, and NRP1, a facilitator of SARS-CoV-2 entry, are expressed in CNS neurons, we hypothesized that SARS-CoV-2 can infect neural cells in the brain, especially under conditions such as Alzheimer's disease and autism with BBB compromise. SARS-CoV2 has been observed in cells of olfactory bulbs. We focused on cognitive centers of the brain. The SARSCoV-2 spike protein and nucleocapsid protein were detected in cells of the inferior frontal cortexes of five COVID-19 cases: two autism cases, one Alzheimer's case, one frontotemporal dementia (FTD) case and one case without any underlying health conditions (apparently healthy case) (Fig. 1, Fig. S1). In contrast, there was no positive staining of spike protein or nucleocapsid protein in the cortexes of the non-COVID19 autism brains (Fig. 1A, C, Fig. S1A). Spike protein- and nucleocapsid protein-positive viral particles were robustly present in the cytoplasm and cellular projections of cortical cells (Fig. 1E, F). RNAscope in situ hybridization detected the abundant presence of SARS-CoV-2 genomic RNA in cortical cells of the five COVID-19 cases (Fig. 1G, H; Fig. S1F). PCR analysis using the CDC method [29] confirmed the presence of SARS-CoV-2 RNA in the three cases that had frozen brain tissues (Fig. 1I). In the 31-yearold COVID-19 autism case, the amounts of SARS-CoV-2 RNA in the inferior frontal cortex and the dorsolateral prefrontal cortex were comparable to those in the lungs (Fig. 1I). In the COVID-19 FTD case and the COVID-19 apparently healthy case, SARS-CoV-2 RNA was also detected, whereas there was no SARS-CoV-2 RNA in the age-matched non-COVID-19 FTD individual or in the age-matched nonCOVID-19 apparently healthy individual (Fig. 1I).

Additional analyses showed that cells of the three cortical regions, the entorhinal cortex, the inferior frontal cortex and the dorsolateral prefrontal cortex, in the two COVID-19 autism cases and the COVID-19 Alzheimer's case possessed abundant spike protein staining signals (Fig. 1J, K, L). The 
numbers of SARS-CoV-2 spike protein-positive cells in these three regions were different in these three

COVID-19 cases (Fig. 1J, K, L). These findings demonstrated that SARS-CoV-2 was neurotropic in this cohort of COVID-19 patients (Table S1).

\section{SARS-CoV-2 infects CNS cells expressing ACE2 and NRP1}

Because SARS-CoV-2 exists in cortical cells in COVID-19 patients, these cells must express ACE2 and NRP1. Indeed, the SARS-CoV-2 spike protein staining signal was colocalized with both ACE2 and

NRP1 signals (Fig. 2A, B), indicating that SARS-CoV-2 infects cortical cells using these proteins. ACE2 neurons (GAD65-positive) and glutamatergic excitatory neurons (glutamine synthetase-positive) 
S2). However, a significant number of these immune cells were detected in the COVID-19 Alzheimer's brain (Fig. S2).

\section{SARS-CoV-2 causes cell death via multiple pathways}

To determine whether SARS-CoV-2-infected cells in the cortical regions undergo cell death, we used immunohistological dual labeling with antibodies against the spike protein and cleaved caspase-3. There

19 autism controls (Fig. 3A). Over 90\% of cleaved caspase 3-positive cells were SARS-CoV-2 spike protein positive in the cortexes of two COVID-19 autism cases (Fig. 3A). In the COVID-19 Alzheimer's disease case, over $20 \%$ of cleaved caspase 3-positive cells were SARS-CoV-2 spike protein positive (Fig.

3B). Cleaved caspase 3 was induced in two COVID-19 autism cases and the COVID-19 case without underlying health conditions and was enhanced in the COVID-19 Alzheimer's case and the COVID-19 FTD case (Fig. S3A, B, C).

To further determine the type of programmed cell death, we examined the presence of necroptotic,

ferroptotic, and senescent cells. The cell necroptosis markers phospho-MLKL (mixed lineage kinase domain-like) and phospho-RIPK3 coexisted in spike protein-positive cells (Fig. 3C, Fig. S3D). Similarly, the two cell ferroptosis markers TfR1 (transferrin receptor) and ASCL4 (long-chain fatty acyl-CoA cytokine associated with SARS-CoV-2 may trigger the cellular senescence program, we detected the senescence marker DPP4 (dipeptidyl-peptidase 4) in a subset of spike protein-positive cells (Fig. 3E). 
senescence through multiple pathways.

\section{SARS-CoV-2 induces neuroinflammation}

It has been suggested that SARS-CoV-2 infection leads to neuroinflammation. However, direct evidence on the link between SARS-CoV-2 and neuroinflammation is still lacking. The protein expression of two cytokines, IL-1 $\beta$ and IL-6 (interleukin 6), was significantly increased in the cortical cells of COVID-19 autism patients compared to the cortical cells of non-COVID-19 autism patients (Fig. S4A, B, C), providing the direct evidence of neuroinflammation induced by SARS-CoV-2.

\section{SARS-CoV-2 induces Alzheimer's-like phenotype development and exacerbation}

Cellular A $\beta$ (amyloid beta) aggregates were observed in the cortexes of the two COVID-19 autism cases (Fig. 4A) and the one COVID-19 case without underlying health conditions (Fig. 4B), whereas it was not present in the cortexes of age-matched non-COVID-19 autism cases and an apparently healthy individual (Fig. 4A, B). Extracellular A $\beta$ plaques were present in one of the COVID-19 autism cases (Fig. 4A). Immunofluorescence analysis with thioflavin-T confirmed that the cytoplasmic deposition of A $\beta$ in the COVID-19 autism cases consisted of aggregated A $\beta$ (Fig. 4A). There was more A $\beta$ plaque deposition per measured area in the cortexes of the COVID-19 Alzheimer's and FTD brains than in the cortexes of age-matched non-COVID-19 Alzheimer's and FTD brains (Fig. 4C, D). One of the neuropathological hallmarks of Alzheimer's disease is the development of intracellular neurofibrillary tangles (NFTs) composed of hyperphosphorylated Tau (microtubule-associated protein tau). p-Tau-containing NFTs are associated with neuronal dysfunction, cognitive deficits and neuronal death [30, 31]. p-Tau-containing NFTs were present in the inferior cortexes of the two COVID-19 autism cases (Fig. 4E). Cellular p-Tau deposition was induced in these two COVID-19 autism cases and the COVID-19 case without underlying health conditions (Fig. 4E, F), whereas there were no signals or negligible p-Tau staining signals in the cortexes of non-COVID-19 control brains (Fig. 4E, F). There were significantly higher numbers of Pick 
bodies in the COVID-19 FTD case than in age-matched non-COVID-19 FTD cases (Fig. 4G, H). Thus,

SARS-CoV-2 infection is linked to Alzheimer's neuropathology.

\section{SARS-CoV-2 infects iPSC-derived mature neurons}

Based on the above findings in neurons of COVID-19 patients' brain cortexes, we propose that SARS-

$\mathrm{CoV}-2$ can effectively infect neurons. To establish an in vitro platform to study this process, we obtained

iPSCs derived from age-matched healthy individuals and Alzheimer's patients and differentiated it into

neurons, followed by SARS-CoV-2 infection. SARS-CoV-2-GFP (in which GFP replaced the viral open

iPSC neuron differentiation day 35 (Fig. 5A, Fig. S5A) and some of these cells expressed the pan-neuron

SARS-CoV-2-GFP could be detected in cell culture media 72 hours post SARS-CoV-2-GFP infection (Fig.

5D), indicating that the virus not only infects cells but also replicates intracellularly. SARS-CoV-2-

GFAT-positive astrocytes were detected in mock- and SARS-CoV-2-infected cells (Fig. 5F). At iPSC infects mature neurons via ACE2 with the facilitation of NRP1.

\section{SARS-CoV-2 induces Alzheimer's phenotypes in iPSC-derived cells}


Alzheimer's neurons (Fig. 6A). Cellular p-Tau deposition was induced in healthy neurons after 72 hours of SARS-CoV-2 infection (Fig. 6B), and the virus further increased cellular p-Tau deposition in Alzheimer's neurons (Fig. 6B). Compared to their mock-infected counterparts, both healthy neurons and TNF $\alpha$ after SARS-CoV-2 infection (Fig. 6C). Among the critical Alzheimer's mediators, amyloid precursor protein (APP), enzyme $\beta$-secretase 1 (BACE1), and presenilin 1/2 (PSEN1/2), SARS-CoV-2 significantly increased BACE1 expression in healthy neurons and Alzheimer's neurons but did not affect the expression of the other Alzheimer's mediators (Fig. 6D, E). Likewise, SARS-CoV-2 significantly increased the number of cleaved caspase 3-positive cells differentiated from iPSCs of healthy individuals and Alzheimer's patients (Fig. 6F). Thus, SARS-CoV-2 triggers an Alzheimer's-like cellular program in neurons derived from iPSCs of healthy individuals and enhances Alzheimer's phenotypes in cells derived from Alzheimer's iPSCs.

\section{Alzheimer's infectious etiology genes identified via SARS-CoV-2}

Over 95\% of Alzheimer's cases are sporadic and their causes are still unclear. Studies of DNA viruses have shown that Alzheimer's etiology has an infectious component [34, 35]. Based on the above observation that SARS-CoV-2 induces Alzheimer's phenotypes in neurons derived from iPSCs of healthy non-Alzheimer's individuals, we aimed to utilize SARS-CoV-2 infection to reveal genes responsible for 
individuals and Alzheimer's patients were determined by RNA sequencing (Fig. 7A-E). Under mock infection conditions, 553 genes were significantly upregulated, while 71 genes were significantly downregulated, in Alzheimer's neurons compared to neurons from iPSCs of healthy individuals (designated healthy neurons) (Fig. 7A). SARS-CoV-2 upregulated 75 genes and downregulated 19 genes in healthy neurons (Fig. 7B). To extract the genes responsible for Alzheimer's infectious etiology, 24 overlapping genes were identified between the Alzheimer's neuron-mock-infected group and the healthy neuron-SARS-CoV-2-infected group (Fig. 7D). Pathway analysis revealed that the changes in these 24 genes activated infection pathways elicited by bacteria and viruses (Fig. 7D).

Compared to healthy neurons without viral infection, Alzheimer's neurons infected with SARS-CoV-2 had 517 upregulated genes and 256 downregulated genes (Fig. 7C). This number of downregulated genes (256) was higher than the number of downregulated genes in Alzheimer's neurons (71) (Fig. 7A, C). In

Alzheimer's neurons, SARS-CoV-2 further increased the expression of 25 upregulated genes and decreased the expression of 34 downregulated genes by several-fold (Fig. S6A), indicating that the virus deteriorates Alzheimer's conditions, and pathway analysis pointed to the further activation of neuroinflammation and other processes in Alzheimer's neurons (Fig. S6B).

\section{Top genes in the Alzheimer's infectious etiology transform neurons}

The 24 overlapping genes between Alzheimer's neurons without SARS-CoV-2 infection and healthy neurons infected by SARS-CoV-2 are potential genes involved in the Alzheimer's infectious etiology. To evaluate whether the top upregulated genes among these 24 genes can turn healthy neurons into

268 Alzheimer's-like neurons, we overexpressed the top three genes individually, FCGR3, LILRB5 and OTOR, in human primary neurons from a heathy individual. Overexpression of these genes in healthy neurons did not trigger cellular A $\beta$ aggregation or cellular p-Tau deposition (Fig. S7D). In contrast, when 
272 silenced in healthy human neurons, cellular expression of $A \beta 42$ and $p$-Tau, cellular $A \beta$ aggregation and

273 cellular p-Tau deposition were induced (Fig. 7F, Fig. S7E). Furthermore, silencing these top three

274 downregulated genes simultaneously in healthy human neurons robustly triggered cellular A $\beta$ aggregation

275 and cellular p-Tau deposition (Fig. 7G). Thus, silencing the top three downregulated genes reprogrammed

276 healthy human neurons into Alzheimer's-like neurons.

277 


\section{Discussion}

Although it is still controversial whether SARS-CoV-2 invades patients' brains $[10,36]$, we provide

strong evidence that SARS-CoV-2 can invade the cognitive centers of the brain, leading to Alzheimer's-

like phenotypes and exacerbation of Alzheimer's neuropathology. Examination revealed that iPSC-

derived neurons infected by SARS-CoV-2 recapitulated the key aspects related to clinically observed

neurological disorders in COVID-19 patients and survivors: neurotropism and Alzheimer's induction or enhancement by SARS-CoV-2.

Our observations from both COVID-19 autism and Alzheimer's brains support the effective

infection of neurons, oligodendrocytes and other brain cells by SARS-CoV-2. Thus, the present study

demonstrates the neurotropism of SARS-CoV-2. Such neurotropism has long been known to occur for

other types of human respiratory coronaviruses [37]. Consistent with the current findings, in human iPSC-

derived brain sphere neurons, SARS-CoV-2 at a dose equivalent to that in the present study has been found

to not only infect neurons but also replicate itself in these neurons [38]. SARS-CoV-2 viral particles are

present in both neuronal cell bodies and neurites [38]. SARS-CoV-2 can effectively infect cortical-like

neurons in iPSC-derived brain organoids and these neurons express the SARS-CoV-2 receptor ACE2 and

key coronavirus entry-associated proteases [39]. Regarding the infectibility of human brains by SARS-

CoV-2, viral RNA has been detected in $36.4 \%$ of brain biopsies of fatal COVID-19 cases [9], suggesting

that SARS-CoV-2 invades some COVID-19 patients' brains but may not invade all patients' brains. The

current study examined cortical neurons of the brains of COVID-19 patients with either autism or

Alzheimer's disease and observed full infectibility by SARS-CoV-2 in all cases. Similarly, a recent study using spike protein staining demonstrated that SARS-CoV-2 was present in some brain regions in all three

COVID-19 cases studied [10]. Using multiple approaches, we showed the presence of spike and 
with either autism or Alzheimer's.

This discrepancy may be due to the different developmental stages of neurons. We show that iPSC-derived

whereas mature neurons do express these two SARS-CoV-2 receptors and are infected by this virus.

Pellegrini et al. [40] did not observe ACE2 expression in neurons. In COVID-19 patients, not all neurons of SARS-CoV-2 into the CNS [8]. The other major CNS entry route may depend on BBB leakage in conditions of autism and Alzheimer's disease $[12,13]$. This may have been the case in our study, in which three COVID-19 patients had either autism or Alzheimer's disease. The BBB entry route is supported by a direct observation of BBB damage in COVID-19 patients [41]. Furthermore, the SARS-CoV-2 spike protein can disrupt human BBB integrity in 3D microfluidic in-vitro models [42]. The infiltration of infected immune cells into the brain has been proposed as a potential CNS entry route [14]. In agreement with a prior report, no sign of infected immune cell infiltration into COVID-19 autism patients' brains was found in the present study. Immune cells were present in the cortex in the COVID-19 Alzheimer's case. 
$43,44]$. SARS-CoV-2-infected cells may not be very susceptible to apoptosis because the majority of cells undergoing apoptosis are noninfected but adjacent to SARS-CoV-2-positive cells [10]. The causes of apoptosis may be SARS-CoV-2-induced cellular inflammation and hypoxia, which occur in brain organoids infected by this virus [10,43]. In COVID-19 patients, we found that apoptosis mostly occurred in cells infected by SARS-CoV-2. We found that cellular inflammation was induced in both iPSC-derived neurons and COVID-19 patient cortexes, suggesting that cellular inflammation is the primary factor leading to cell death. Other factors such as p-Tau, as detected by our group and others [44], may also play important roles in defining cell fates. SARS-CoV-2 infection induces not only apoptosis but also other nonapoptotic cell death programs, such as necroptosis and ferroptosis, as well as senescence [45], as observed in the current study. Thus, SARS-CoV-2 infection alters cellular programs, leading to critical cell mass loss and cellular dysfunction.

COVID-19 patients and survivors experience Alzheimer's-like neural syndrome including memory loss, delirium and cognitive deficits [25], suggesting that there is a cellular mechanism underlying these phenomena. A prior study showed altered distribution of Tau from axons to soma and Tau hyperphosphorylation in neurons of brain organoids exposed to SARS-CoV-2 [44]. We observed p-Tau tangles in the cortexes of two young autism COVID-19 patients, suggesting that neurodegenerative characteristics manifest in COVID-19 patients' brains. A $\beta$ deposition and neuroinflammation were also present in the cognitive centers of these two young autism COVID-19 patients and in iPSC-derived neurons infected by SARS-CoV-2. These observations potentially explain the Alzheimer's-like brain disease observed in individuals exposed to SARS-CoV-2.

The pathogenesis of Alzheimer's disease contains an element of infectious disease pathogenesis. Pathogen infections can lead to the onset and progression of Alzheimer's disease. Insertions of viral DNA genomes into spontaneous late-onset Alzheimer's patient genomes have been determined [34]. Viral DNA 
insertions in the host genome correlate with the induction of critical Alzheimer's mediators, such as enzymes involved in A $\beta$ species production, aggregation and plaque formation [34]. Direct evidence for the involvement of viral DNA in Alzheimer's pathogenesis comes from the demonstration that herpes simplex virus type I (HSV-1) induces multicellular amyloid plaque-like structure formation, gliosis, and neuroinflammation in iPSC-derived neural cells and a 3D human brain-like model [35]. We provide direct evidence that the COVID-19 pandemic-causing virus SARS-CoV-2 triggers an Alzheimer's-like molecular program involving a group of 24 genes related to infectious disease pathway activation. The $\beta$ secretase BACE1, which produces all monomeric forms of amyloid- $\beta(A \beta)$, including $A \beta 42$, was induced by SARS-CoV-2 in iPSC-derived neurons from healthy individuals; thus, BACE1 may be a primary driver of the Alzheimer's-like phenotypes induced by SARS-CoV-2. SARS-CoV-2-induced hypoxia [10] may be responsible for BACE1 induction because hypoxia facilitates Alzheimer's pathogenesis by inducing BACE1 expression [46]. Because 26 of the 29 SARS-CoV-2 proteins physically associate with many proteins in human cells [47], it is also possible that one or some of the 29 SARS-CoV-2 proteins interact with transcriptional regulators in host cells, leading to BACE1 upregulation. The mechanism underlying SARS-CoV-2-induced Alzheimer's-like phenotypes needs to be further investigated. In summary, we found that SARS-CoV-2 neurotropism exhibited full penetrance in the cortexes of COVID-19 autism and Alzheimer's patients. SARS-CoV-2 infection induced Alzheimer's-like phenotypes in autism patients and exacerbated neuropathology in Alzheimer's patients. SARS-CoV-2 infection triggered cellular and molecular Alzheimer's pathogenesis programs in iPSC-derived neurons

366 from healthy individuals and enhanced neuropathological phenotypes in iPSC-derived neurons from 367 Alzheimer's patients. We reveal a list of 24 genes that potentially mediate the infectious etiology of 


\section{Acknowledgments}

371 This work was supported by NIH grants R01HD100195, R01HD102206, R01HD099843, R01DK083243,

372 R01DK101972, R01HL131737, R01HL134368, R01HL139060, R01DK103024 and P30-AG062429. No

373 potential conflicts of interest relevant to this article are reported. The authors thank Jeffrey Metcalf, Sara

374 Shuldberg and Sophia Perrott from the Rissman lab and UCSD Shiley-Marcos ADRC for technical 375 assistance with this project.

376

\section{Author Contributions}

Shen WB, Penghua Yang, Montasir M, Xu C, Logue J, and Baracco L researched the data. Frieman

M, Reece EA, Blanchard T, Li L, Han Z, and Rissman R analyzed the data and revised the manuscript. approved the final version of the paper.

\section{Declaration of Interests}

The authors declare no competing interests. 


\section{Main figure titles and legends}

387

388

389

390

391

392

393

394

395

396

397

398

399

400

401

402

403

404

405

406

407

408

\section{Figure 1. SARS-CoV-2 is present in cortical cells in COVID-19 patients.}

Representative images of SARS-CoV-2 spike protein staining in the inferior frontal cortexes of an agematched non-COVID-19 autism (ASD) case (A) and a COVID-19 autism case (ID \#6437) (B).

Representative images of SARS-CoV-2 nucleocapsid protein in the inferior frontal cortexes of an agematched non-COVID-19 autism case (C) and a COVID-19 autism case (ID \#6437) (D).

High-magnification imaging indicated that spike protein-positive (E) or nucleocapsid protein-positive (F) viral particles were located in both the cytoplasm and cellular processes (arrows).

SARS-CoV-2 genomic RNA was detected by RNAscope in the cortical cells of the COVID-19 autism case (H, arrows) but not in the age-matched non-COVID-19 autism case (G).

COVID-19 PCR test revealed SARS-CoV-2 positivity in brain regions of two COVID-19 cases along with lung positivity (ID \#6436) (I). Red * indicates nonspecific bands compared to the 72 bp positive band. Melt curve analysis and DNA sequencing of PCR products confirmed SARS-CoV-2 positivity in brain tissues.

SARS-CoV-2 spike protein staining positivity in the EC, IFC and DPC in the COVID-19 Alzheimer's case (ID \#6435) (J) and in COVID-19 autism case 1 (ID \#6436) (K) and case 2 (ID \#6437) (L). The spike protein-positive cells per $\mathrm{mm}^{2}$ were quantified in the three brain regions. Cells were counted from at least 3 sections ( $\mathrm{n} \geq 3$ ). EC: Entorhinal cortex; IFC: inferior frontal cortex; DPC: dorsolateral prefrontal cortex. Scale bars: A-D, J-L $=20 \mu \mathrm{m} ; \mathrm{E}, \mathrm{F}=10 \mu \mathrm{m} ; \mathrm{G}, \mathrm{H}=5 \mu \mathrm{m} . *$ indicate significant difference $(P<0.05)$ by one-way ANOVA and Tukey test.

Figure 2. hACE2-positive or neuropilin 1 (NRP1)-positive neurons and nonneuronal cells exhibit SARS-CoV-2 positivity.

(A) Double spike protein (SP, Ni-DAB: black signals)- and hACE2 (AP staining: red signals)-positive 

case and their age-matched control cases: non-COVID-19 autism control cases and non-COVID-19

411 Alzheimer's cases. The graphs show the percentages of double positive cells among total hACE2 positive 412 cells in $\mathrm{n} \geq 5$ random fields.

(B) Double SP (Ni-DAB: black signals)- and NRP1 (AP staining: red signals)-positive cells in the inferior frontal cortexes of the COVID-19 autism cases, the COVID-19 Alzheimer's case and their age-matched control cases. The graphs show the percentages of double positive cells among total NRP1-positive cells in $\mathrm{n} \geq 5$ random fields.

(C) Controls for immunohistological staining with normal IgG for Ni-DAB staining, AP staining or both in the inferior frontal cortexes (IFCs) of non-COVID-19 autism cases and with an hACE2 antibody for Ni-DAB staining.

Detection of the hACE2 receptor in neurons and nonneuronal cells in the IFCs of non-COVID-19 autism cases. The hACE2-positive neurons are labeled with antibodies against NFL (D) or Tuj1 (E), the oligodendrocyte marker CNPase (F), and the microglial marker Iba1 (G).

(H) hACE2 and cell marker double-positive cells were quantified ( $\%, \mathrm{n} \geq 5$ random fields).

SP positivity was detected in neurons and nonneuronal cells in the IFCs of the COVID-19 autism case (ID \#6437). SP-positive neurons labeled with Tuj1 (I), NeuN (J), the GABAergic inhibitory neuronal marker GAD65 (K), the glutamatergic excitatory neuronal marker GS (glutamine synthetase) (L), the oligodendrocyte marker CNPase (M), and the microglial marker Iba1 (N). GFAP ${ }^{+}$astrocytes were SP negative (green arrows) $(\mathbf{O})$.

(P) Quantification of SP and cell marker double positive cells ( $\%, \mathrm{n} \geq 5$ random fields). 
Figure 3. Spike protein-positive cells undergo programmed cell death.

Representative images of double SARS-CoV-2 spike protein (SP) (Ni-DAB staining: black signal) and cleaved caspase 3 (cl-casp3; AP: red signal) staining in the cortexes of age-matched non-COVID-19 autism and COVID-19 autism cases (A) and non-COVID-19 Alzheimer's and the COVID-19 Alzheimer's case (B). The inset boxes show cl-casp3 and SP double-labeled cells, cl-casp3-positive cells and doublenegative cells.

The graphs show the percentages of double $\mathrm{SP}^{+}$and cl-casp $3^{+}$cells among total cleaved caspase $3^{+}$cells in at least 5 random fields. * indicate significant difference $(P<0.05)$ compared with the non-COVID-19 controls by $t$ test.

Representative images of SP staining with the necroptosis marker phospho-MLKL (C), the ferroptosis marker TfR1 (D), and the senescence marker DPP4 (E) in the cortexes of the non-COVID-19 autism cases, the COVID-19 Alzheimer's case and the two COVID-19 autism cases. Black arrows point to the double $\mathrm{SP}^{+}$and cl-casp3 ${ }^{+}$cells.

The graphs show the percentages of $\mathrm{SP}^{+}$necroptotic cells, ferroptotic cells and senescent cells counted from at least 5 random fields ( $\mathrm{n} \geq 5$, in 2 COVID-19 ASD cases and 1 COVID-19 AD case). * indicate significant difference $(P<0.05)$ compared with the non-COVID-19 controls by one-way ANOVA and Tukey test. Scale bars $=20 \mu \mathrm{m}$.

\section{Figure 4. SARS-CoV-2 infection induces or enhances $A \beta$ aggregate/plaque and $p$-Tau pretangles}

\section{formation.}

$\mathrm{A} \beta$ immunohistochemical staining (with the anti-A $\beta$ antibody $6 \mathrm{E} 10$ ) in three cortical regions of two cases of COVID-19 autism (A) and one case of COVID-19 with no underlying conditions (NUC) (B) and their age-matched control cases. Cytoplasmic deposition of A $\beta$ (blue arrows) was observed in COVID-19 
autism (ASD) cortexes, and A $\beta$ plaques (red arrow) were observed in the EC of one COVID-19 ASD case. immunohistochemical staining in the cortical regions and hippocampi of the COVID-19 FTD and in ASD and NUC cases or $\mathrm{A} \beta$ plaques per $\mathrm{mm}^{2}$ in FTD and $\mathrm{AD}$ cases $(3$ sections for each region and 3 independent images for one section). (E) p-Tau (pSer202) staining in the cortexes of COVID-19 ASD (E) and COVID-19 NUC (F) cases and their age-matched controls. (G) p-Tau staining in the cortexes and hippocampi of the two COVID-19 FTD and Alzheimer's disease cases and their age-matched controls. tangle numbers in AD cases. Entorhinal cortex: EC; inferior frontal cortex: IFC; dorsolateral prefrontal distribution). Bars $=100 \mu \mathrm{m}$.

Figure 5. SARS-CoV-2 infects mature neurons derived from human iPSCs.

(A). SARS-CoV-2-GFP does not infect immature neurons at iPSC neuron differentiation day 35 ( $\mathrm{n}=3$ replicates).

474 (B). Immature neurons do not express the mature neuron marker, NeuN.

(C). SARS-CoV-2 infects mature neurons at iPSC neuron differentiation day $50(\mathrm{n}=3)$.

(D). Detection of SARS-CoV-2 in the culture medium of infected neurons $(n=3)$.

(E). SARS-CoV-2 infects Tuj1-positive neurons $(\mathrm{n}=3)$. 
(F). GFAP is not expressed in SARS-CoV-2-infected cells at iPSC differentiation day $50(\mathrm{n}=2)$.

Immature Tuj1-positive neurons do not express ACE2 (G) and NRP1 (H) $(\mathrm{n}=3)$.

Mature Tuj1 positive neurons express ACE2 (I) and NRP1 (J) $(\mathrm{n}=3)$.

The bar graphs show the percentages of double Tuj1- and ACE2- or NRP1-positive cells (yellow) among spike protein.

Figure 6. SARS-CoV-2 induces or enhances Alzheimer's-like neuron phenotypes. and Alzheimer's patients ( $\mathrm{n}=3$ iPSC lines).

(B). SARS-CoV-2 induces cellular p-Tau deposition in neurons derived from iPSCs of healthy individuals and Alzheimer's patients ( $\mathrm{n}=3$ iPSC lines).

The values in the graphs are presented as the means \pm SEMs $(n=5$ images from 2 independent experiments for each group). The $\mathrm{A} \beta$ and $\mathrm{p}$-Tau intensity data were analyzed by Mann-Whitney $\mathrm{U}$ test after confirming a normal distribution. Bars $=50 \mu \mathrm{m}$.

mRNAs levels of inflammatory cytokines (C), BACE1 (D), and Alzheimer's molecular mediators (APP, PSEN1 and PSEN2) (E) in SARS-CoV-2-infected neurons derived from iPSCs ( $\mathrm{n}=6,3$ iPSC lines for 2 independent experiments).

F. Cleaved caspase 3 (c-Cas3)-positive cells in SARS-CoV-2 infected neurons derived from iPSCs ( $\mathrm{n}=6$ iPSC lines for 2 independent experiments). The bar graph shows the quantification of c-Cas3-positive cell numbers. CTL: control group (iPSCs from healthy individuals); AD: Alzheimer's disease; CTLv: SARS- 
501 significant $P$-values are presented.

502 Figure 7. Identification of an infectious etiology gene signature for Alzheimer's disease.

503 (A). Volcano plot of differentially expressed genes (DEGs) in neurons derived from iPSCs of patients with

504 Alzheimer's disease (AD) (the AD group) compared to neurons derived from iPSCs of healthy individuals

505 (the CTL group).

506 (B). Volcano plot of DEGs in healthy individual iPSC-derived neurons infected with SARS-CoV-2 (the

507 CTL_V group) compared to the CTL group.

508 (C). Volcano plot of DEGs in AD neurons infected with SARS-CoV-2 (the AD_V group) compared to the 509 CTL group.

510 (D). Venn plot and KEGG pathway analysis for DEGs between the AD group and the CTL_V group. The

511 CTL group served as the baseline group. The twenty-four overlapping genes constituted the AD infectious 512 etiology gene signature.

513 (E). Venn plot and KEGG pathway analysis for DEGs between the AD group and the AD_V group. The 514 CTL group served as the baseline group.

515 For $\mathbf{A}$ to $\mathbf{E}, \mathrm{n}=3$ iPSC lines for the CTL and AD groups.

516 (F-G). Analysis of the effect of siRNA mediated individual or combined knockdown of the top 3

517 downregulated genes, GJA8, CryAA2 and PSG6, on the AD infectious etiology gene signature in primary

518 human neurons. F: Immunoblot analysis of $A \beta$ isoforms $(n=3$ replicates). G. Cellular A $\beta$ aggregation 519 (left panel) and cellular p-Tau deposition (right panel). A $\beta$ aggregates and p-Tau accumulation in the 520 cytoplasm or neuronal axon are indicated by white arrowheads. The values in the graphs are presented as 521 the means \pm SEMs ( $\mathrm{n}=8$ images from 3 replicates). Intensity data were analyzed by Mann-Whitney $\mathrm{U}$ 522 test after confirming a normal distribution, significant $P$-values are presented. Scale bar, $50 \mu \mathrm{m}$. 


\section{Supplemental figure titles and legends}

\section{Fig. S1. Presence of SARS-CoV-2 spike protein in cortical cells of five COVID-19 cases.}

526 Spike protein staining signals were visualized with an alkaline phosphatase (AP)-coupled goat anti-rabbit

527

secondary antibody and AP red substrate. SARS-CoV-2 spike protein red signals were located in the cytosol of cortical cells of one COVID-19 autism (ASD) case but not in cells of an age-matched nonCOVID-19 ASD control (A). Spike protein (DAB staining, brown signal; counterstained with hematoxylin, blue) was present in the cytosol of cortical cells of the COVID-19 FTD case (ID \#5922) (C, three different fields, arrows) but not in those of the age-matched non-COVID-19 FTD controls (B, 3 cases). In the COVID-19 case without underlying conditions (ID \#5932), the spike protein was also visible (E, three different fields, arrows), but it was not visible in the age-matched non-COVID-19 AD controls (D, 3 cases). RNAscope in situ hybridization showing COVID-19 genomic RNAs in four COVID-19 cases (F): 2 ASD COVID-19 cases, 1 AD COVID-19 case, 1 FTD COVID-19 case, and 1 COVID-19 case without underlying conditions. Scale bar $=20 \mu \mathrm{m}$.

\section{Fig. S2. Immunostaining of immune cell markers in brain tissues of COVID-19 patients.}

Immunolabeling (DAB, brown signal) with antibodies against CD3 (T cell marker, A), CD20 (B cell marker, B), and F4/80 (macrophage marker, C) showed positively labeled immune cells in the cortex of the COVID-19 Alzheimer's case. Immune cells were not detected in non-COVID-19 autism cases or the two COVID-19 autism cases (A-C). Counterstaining: hematoxylin. The arrows point to immune cells. (D) The percentages of immune cells were quantified from 3 sections $(n=3)$. ${ }^{*}$ indicates a significant difference $(P<0.05)$ by one-way ANOVA and Tukey's test. Scale bar $=20 \mu \mathrm{m}$.

\section{Fig. S3. Programmed cell death in SARS-CoV-2 spike protein positive-cortical cells.}

Representative images of cleaved caspase-3 staining (DAB, brown signal) in the cortexes of age-matched 
non-COVID-19 autism cases, 2 COVID-19 autism cases, and 1 COVID-19 case with no underlying

Alzheimer's, non-COVID-19 FTD, and COVID-19 FTD cases (B). Counterstaining: hematoxylin (blue). necroptotic cells (D) and ASCL4 ${ }^{+}$ferroptotic cells (E) in the non-COVID-19, COVID-19 Alzheimer's

of double-positive cell numbers (spike protein and programmed cell death marker positive) from at least

5 random fields $(\mathrm{n} \geq 5)$ * indicates a significant difference $(P<0.05)$ compared with non-COVID-19 controls by one-way ANOVA and Tukey's test. Scale bar $=20 \mu \mathrm{m}$.

Figure. S4. Cytokine expression in COVID-19 autism cortexes.

COVID-19 autism (ASD) and in age-matched non-COVID-19 ASD controls (CTL) in the entorhinal cortex (EC). (C) Quantification of immunostaining intensity ( $n=6,3$ random fields per case per region). is presented. Scale bar $=100 \mu \mathrm{m}$.

Fig. S5. SARS-CoV-2 infects mature neurons derived from human iPSCs.

568 (A) SARS-CoV-2-GFP does not infect immature neurons at iPSC differentiation day 35. White asterisks

(*) indicate nonspecific fluorescent signals. (B) Immature neurons do not express the mature neuron 
individuals): control groups; AD: Alzheimer's disease; Tuj1: beta-Tubulin III; SPIKE: SARS-CoV-2 spike protein. group, $n=3$.

(D) Immunofluorescence staining with the indicated antibodies after lentivirus-mediated overexpression and only FCGR overexpression increased Tau phosphorylation at Ser202. 


\section{References}

592 [1] Helms J, Kremer S, Merdji H, Clere-Jehl R, Schenck M, Kummerlen C, et al. Neurologic Features in Severe SARS-CoV-2 593 Infection. N Engl J Med. 2020;382:2268-70.

594 [2] Mao L, Jin H, Wang M, Hu Y, Chen S, He Q, et al. Neurologic Manifestations of Hospitalized Patients With Coronavirus 595 Disease 2019 in Wuhan, China. JAMA Neurol. 2020;77:683-90.

596 [3] de Erausquin GA, Snyder H, Carrillo M, Hosseini AA, Brugha TS, Seshadri S, et al. The chronic neuropsychiatric sequelae of COVID-19: The need for a prospective study of viral impact on brain functioning. Alzheimers Dement. 2021.

598 [4] Taquet M, Geddes JR, Husain M, Luciano S, Harrison PJ. 6-month neurological and psychiatric outcomes in 236379 599 survivors of COVID-19: a retrospective cohort study using electronic health records. Lancet Psychiatry. 2021;8:416-27.

600 [5] De Santis G. SARS-CoV-2: A new virus but a familiar inflammation brain pattern. Brain Behav Immun. 2020;87:95-6.

601 [6] Li K, Wohlford-Lenane C, Perlman S, Zhao J, Jewell AK, Reznikov LR, et al. Middle East Respiratory Syndrome 602 Coronavirus Causes Multiple Organ Damage and Lethal Disease in Mice Transgenic for Human Dipeptidyl Peptidase 4. J 603 Infect Dis. 2016;213:712-22.

604 [7] Netland J, Meyerholz DK, Moore S, Cassell M, Perlman S. Severe acute respiratory syndrome coronavirus infection causes 605 neuronal death in the absence of encephalitis in mice transgenic for human ACE2. J Virol. 2008;82:7264-75.

606 [8] Meinhardt J, Radke J, Dittmayer C, Franz J, Thomas C, Mothes R, et al. Olfactory transmucosal SARS-CoV-2 invasion as 607 a port of central nervous system entry in individuals with COVID-19. Nat Neurosci. 2021;24:168-75.

608 [9] Puelles VG, Lutgehetmann M, Lindenmeyer MT, Sperhake JP, Wong MN, Allweiss L, et al. Multiorgan and Renal Tropism 609 of SARS-CoV-2. N Engl J Med. 2020;383:590-2.

610 [10] Song E, Zhang C, Israelow B, Lu-Culligan A, Prado AV, Skriabine S, et al. Neuroinvasion of SARS-CoV-2 in human and 611 mouse brain. J Exp Med. 2021;218.

612 [11] Zheng J, Wong LR, Li K, Verma AK, Ortiz ME, Wohlford-Lenane C, et al. COVID-19 treatments and pathogenesis 613 including anosmia in K18-hACE2 mice. Nature. 2021;589:603-7.

614 [12] Montagne A, Zhao Z, Zlokovic BV. Alzheimer's disease: A matter of blood-brain barrier dysfunction? J Exp Med. $615 \quad 2017 ; 214: 3151-69$.

616 [13] Fiorentino M, Sapone A, Senger S, Camhi SS, Kadzielski SM, Buie TM, et al. Blood-brain barrier and intestinal epithelial 617 barrier alterations in autism spectrum disorders. Mol Autism. 2016;7:49.

618 [14] Iadecola C, Anrather J, Kamel H. Effects of COVID-19 on the Nervous System. Cell. 2020;183:16-27 e1.

619 [15] Hoffmann M, Kleine-Weber H, Schroeder S, Kruger N, Herrler T, Erichsen S, et al. SARS-CoV-2 Cell Entry Depends on 620 ACE2 and TMPRSS2 and Is Blocked by a Clinically Proven Protease Inhibitor. Cell. 2020;181:271-80 e8.

621 [16] Butowt R, Bilinska K. SARS-CoV-2: Olfaction, Brain Infection, and the Urgent Need for Clinical Samples Allowing 622 Earlier Virus Detection. ACS Chem Neurosci. 2020;11:1200-3.

623 [17] Cantuti-Castelvetri L, Ojha R, Pedro LD, Djannatian M, Franz J, Kuivanen S, et al. Neuropilin-1 facilitates SARS-CoV-2 cell entry and infectivity. Science. 2020;370:856-60.

[18] Vanacker C, Trova S, Shruti S, Casoni F, Messina A, Croizier S, et al. Neuropilin-1 expression in GnRH neurons regulates prepubertal weight gain and sexual attraction. EMBO J. 2020;39:e104633.

627 [19] Rogers JP, Chesney E, Oliver D, Pollak TA, McGuire P, Fusar-Poli P, et al. Psychiatric and neuropsychiatric presentations 628 associated with severe coronavirus infections: a systematic review and meta-analysis with comparison to the COVID-19 629 pandemic. Lancet Psychiatry. 2020;7:611-27.

630 [20] Varatharaj A, Thomas N, Ellul MA, Davies NWS, Pollak TA, Tenorio EL, et al. Neurological and neuropsychiatric complications of COVID-19 in 153 patients: a UK-wide surveillance study. Lancet Psychiatry. 2020;7:875-82.

[21] Paterson RW, Brown RL, Benjamin L, Nortley R, Wiethoff S, Bharucha T, et al. The emerging spectrum of COVID-19 neurology: clinical, radiological and laboratory findings. Brain. 2020;143:3104-20.

634 [22] Kadono Y, Nakamura Y, Ogawa Y, Yamamoto S, Kajikawa R, Nakajima Y, et al. A case of COVID-19 infection presenting 
[23] Hosseini AA, Shetty AK, Sprigg N, Auer DP, Constantinescu CS. Delirium as a presenting feature in COVID-19: Neuroinvasive infection or autoimmune encephalopathy? Brain Behav Immun. 2020;88:68-70.

638 [24] Alkeridy WA, Almaghlouth I, Alrashed R, Alayed K, Binkhamis K, Alsharidi A, et al. A Unique Presentation of Delirium in a Patient with Otherwise Asymptomatic COVID-19. J Am Geriatr Soc. 2020;68:1382-4.

640 [25] Jaywant A, Vanderlind WM, Alexopoulos GS, Fridman CB, Perlis RH, Gunning FM. Frequency and profile of objective cognitive deficits in hospitalized patients recovering from COVID-19. Neuropsychopharmacology. 2021.

642 [26] Heneka MT, Golenbock D, Latz E, Morgan D, Brown R. Immediate and long-term consequences of COVID-19 infections 643 for the development of neurological disease. Alzheimers Res Ther. 2020;12:69.

644 [27] Vallamkondu J, John A, Wani WY, Ramadevi SP, Jella KK, Reddy PH, et al. SARS-CoV-2 pathophysiology and 645 assessment of coronaviruses in CNS diseases with a focus on therapeutic targets. Biochim Biophys Acta Mol Basis Dis. $646 \quad 2020 ; 1866: 165889$.

647 [28] Wang Q, Davis PB, Gurney ME, Xu R. COVID-19 and dementia: Analyses of risk, disparity, and outcomes from electronic 648 health records in the US. Alzheimers Dement. 2021.

649 [29] Coil DA, Albertson T, Banerjee S, Brennan G, Campbell AJ, Cohen SH, et al. SARS-CoV-2 detection and genomic sequencing from hospital surface samples collected at UC Davis. PLoS One. 2021;16:e253578.

651 [30] Gomez-Isla T, Hollister R, West H, Mui S, Growdon JH, Petersen RC, et al. Neuronal loss correlates with but exceeds neurofibrillary tangles in Alzheimer's disease. Ann Neurol. 1997;41:17-24.

653 [31] Giannakopoulos P, Herrmann FR, Bussiere T, Bouras C, Kovari E, Perl DP, et al. Tangle and neuron numbers, but not amyloid load, predict cognitive status in Alzheimer's disease. Neurology. 2003;60:1495-500.

655 [32] Hou YJ, Okuda K, Edwards CE, Martinez DR, Asakura T, Dinnon KH, 3rd, et al. SARS-CoV-2 Reverse Genetics Reveals a Variable Infection Gradient in the Respiratory Tract. Cell. 2020;182:429-46 e14.

657 [33] Tian JH, Patel N, Haupt R, Zhou H, Weston S, Hammond H, et al. SARS-CoV-2 spike glycoprotein vaccine candidate NVX-CoV2373 immunogenicity in baboons and protection in mice. Nat Commun. 2021;12:372.

659 [34] Readhead B, Haure-Mirande JV, Funk CC, Richards MA, Shannon P, Haroutunian V, et al. Multiscale Analysis of Independent Alzheimer's Cohorts Finds Disruption of Molecular, Genetic, and Clinical Networks by Human Herpesvirus. Neuron. 2018;99:64-82 e7.

662 [35] Cairns DM, Rouleau N, Parker RN, Walsh KG, Gehrke L, Kaplan DL. A 3D human brain-like tissue model of herpesinduced Alzheimer's disease. Sci Adv. 2020;6:eaay8828.

664 [36] Thakur KT, Miller EH, Glendinning MD, Al-Dalahmah O, Banu MA, Boehme AK, et al. COVID-19 neuropathology at 665 Columbia University Irving Medical Center/New York Presbyterian Hospital. Brain. 2021.

666 [37] Arbour N, Day R, Newcombe J, Talbot PJ. Neuroinvasion by human respiratory coronaviruses. J Virol. 2000;74:8913-21. 667 [38] Bullen CK, Hogberg HT, Bahadirli-Talbott A, Bishai WR, Hartung T, Keuthan C, et al. Infectability of human BrainSphere 668 neurons suggests neurotropism of SARS-CoV-2. ALTEX. 2020;37:665-71.

669 [39] Zhang BZ, Chu H, Han S, Shuai H, Deng J, Hu YF, et al. SARS-CoV-2 infects human neural progenitor cells and brain 670 organoids. Cell Res. 2020;30:928-31.

671 [40] Pellegrini L, Albecka A, Mallery DL, Kellner MJ, Paul D, Carter AP, et al. SARS-CoV-2 Infects the Brain Choroid Plexus 672 and Disrupts the Blood-CSF Barrier in Human Brain Organoids. Cell Stem Cell. 2020;27:951-61 e5.

673 [41] Alexopoulos H, Magira E, Bitzogli K, Kafasi N, Vlachoyiannopoulos P, Tzioufas A, et al. Anti-SARS-CoV-2 antibodies in the CSF, blood-brain barrier dysfunction, and neurological outcome: Studies in 8 stuporous and comatose patients. Neurol Neuroimmunol Neuroinflamm. 2020;7.

676 [42] Buzhdygan TP, DeOre BJ, Baldwin-Leclair A, Bullock TA, McGary HM, Khan JA, et al. The SARS-CoV-2 spike protein alters barrier function in 2D static and 3D microfluidic in-vitro models of the human blood-brain barrier. Neurobiol Dis. 2020;146:105131.

679 [43] Jacob F, Pather SR, Huang WK, Zhang F, Wong SZH, Zhou H, et al. Human Pluripotent Stem Cell-Derived Neural Cells 680 and Brain Organoids Reveal SARS-CoV-2 Neurotropism Predominates in Choroid Plexus Epithelium. Cell Stem Cell. 
682 [44] Ramani A, Muller L, Ostermann PN, Gabriel E, Abida-Islam P, Muller-Schiffmann A, et al. SARS-CoV-2 targets neurons 683 of 3D human brain organoids. EMBO J. 2020;39:e106230.

684 [45] Lee S, Yu Y, Trimpert J, Benthani F, Mairhofer M, Richter-Pechanska P, et al. Virus-induced senescence is driver and 685 therapeutic target in COVID-19. Nature. 2021.

686 [46] Sun X, He G, Qing H, Zhou W, Dobie F, Cai F, et al. Hypoxia facilitates Alzheimer's disease pathogenesis by up-regulating 687 BACE1 gene expression. Proc Natl Acad Sci U S A. 2006;103:18727-32.

688 [47] Gordon DE, Jang GM, Bouhaddou M, Xu J, Obernier K, White KM, et al. A SARS-CoV-2 protein interaction map reveals 689 targets for drug repurposing. Nature. 2020;583:459-68.

690 
Fig. 1
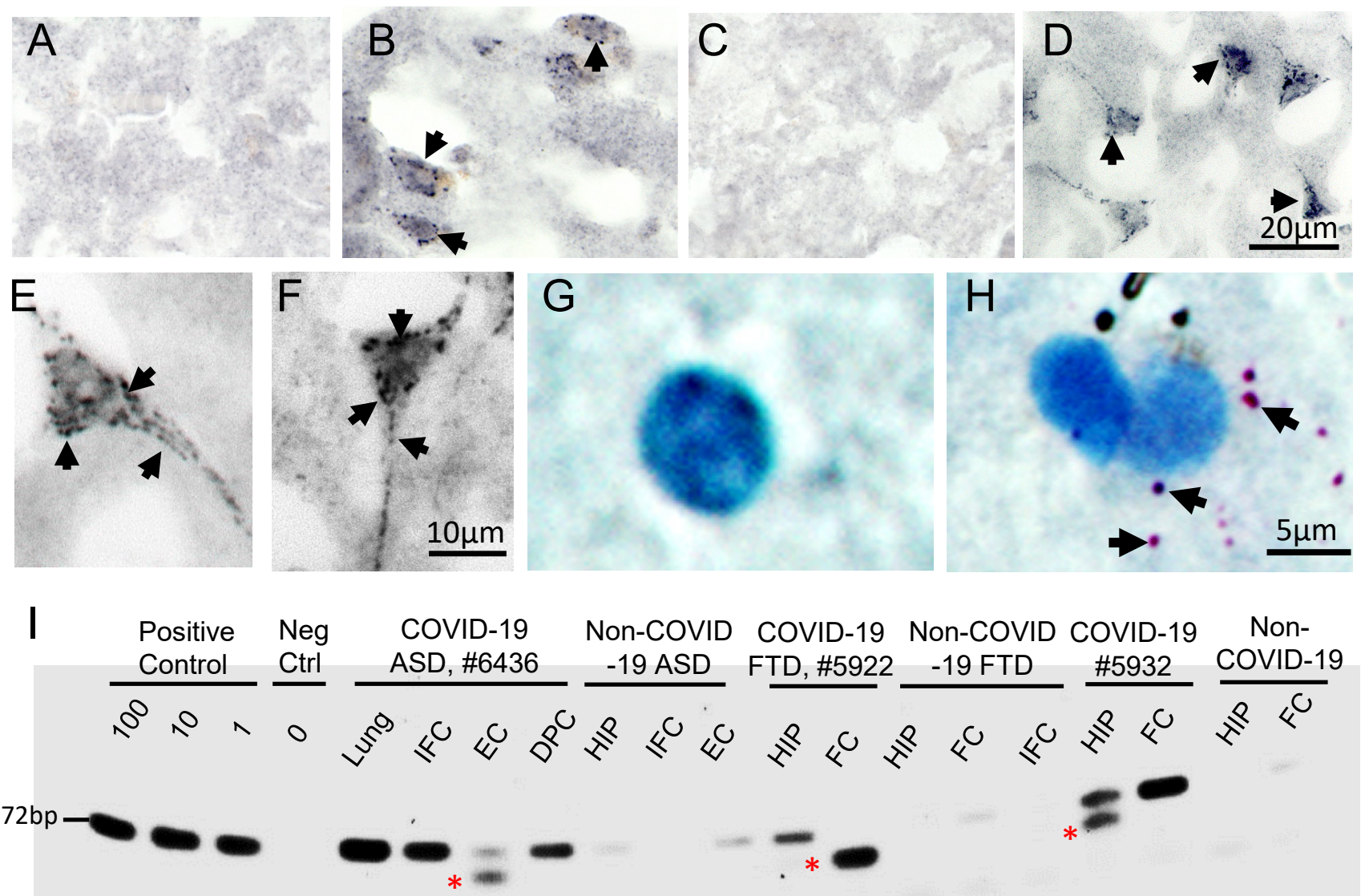

$\beta$-actin

EC

J

\#6435

$\mathrm{K}$

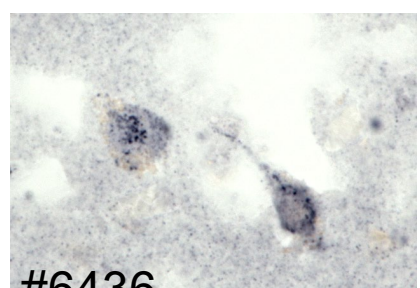

\#6436

L

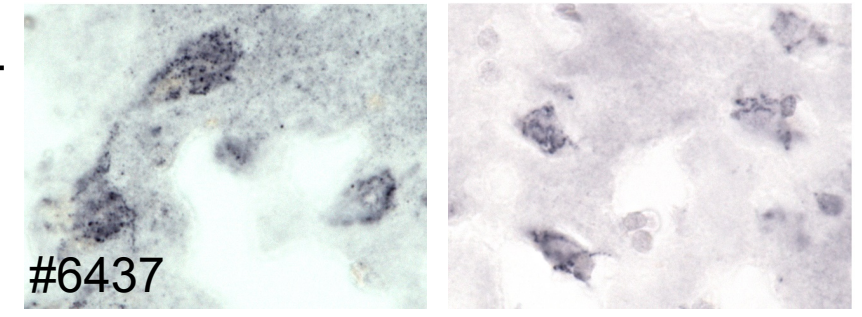

IFC
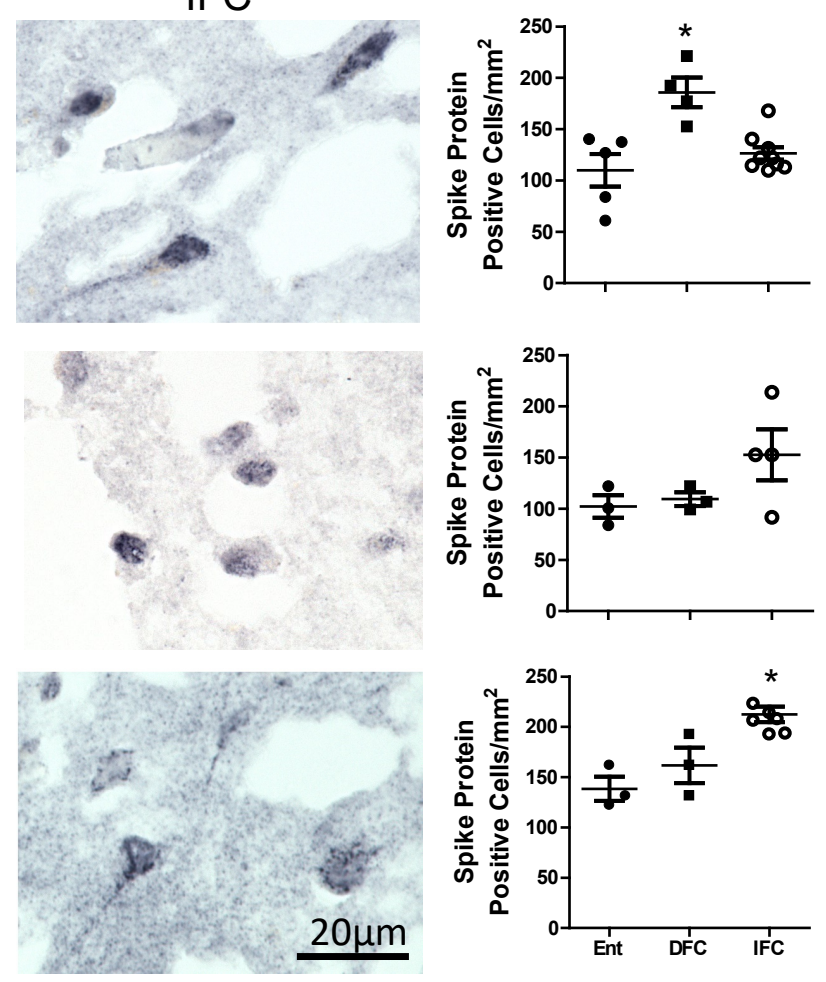

产

竞竞 50

Ent DFC IFC 
Fig. 2

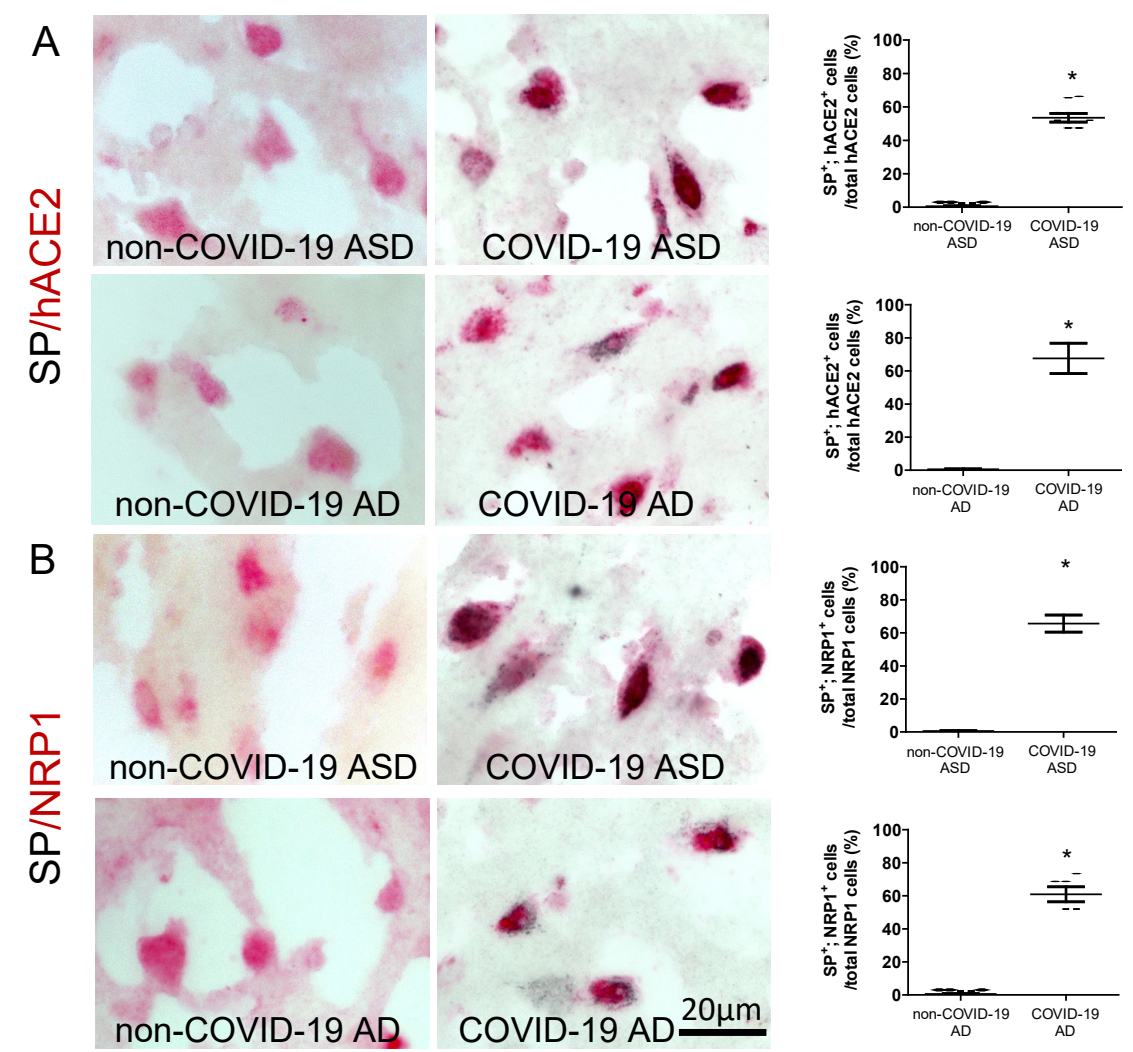

C

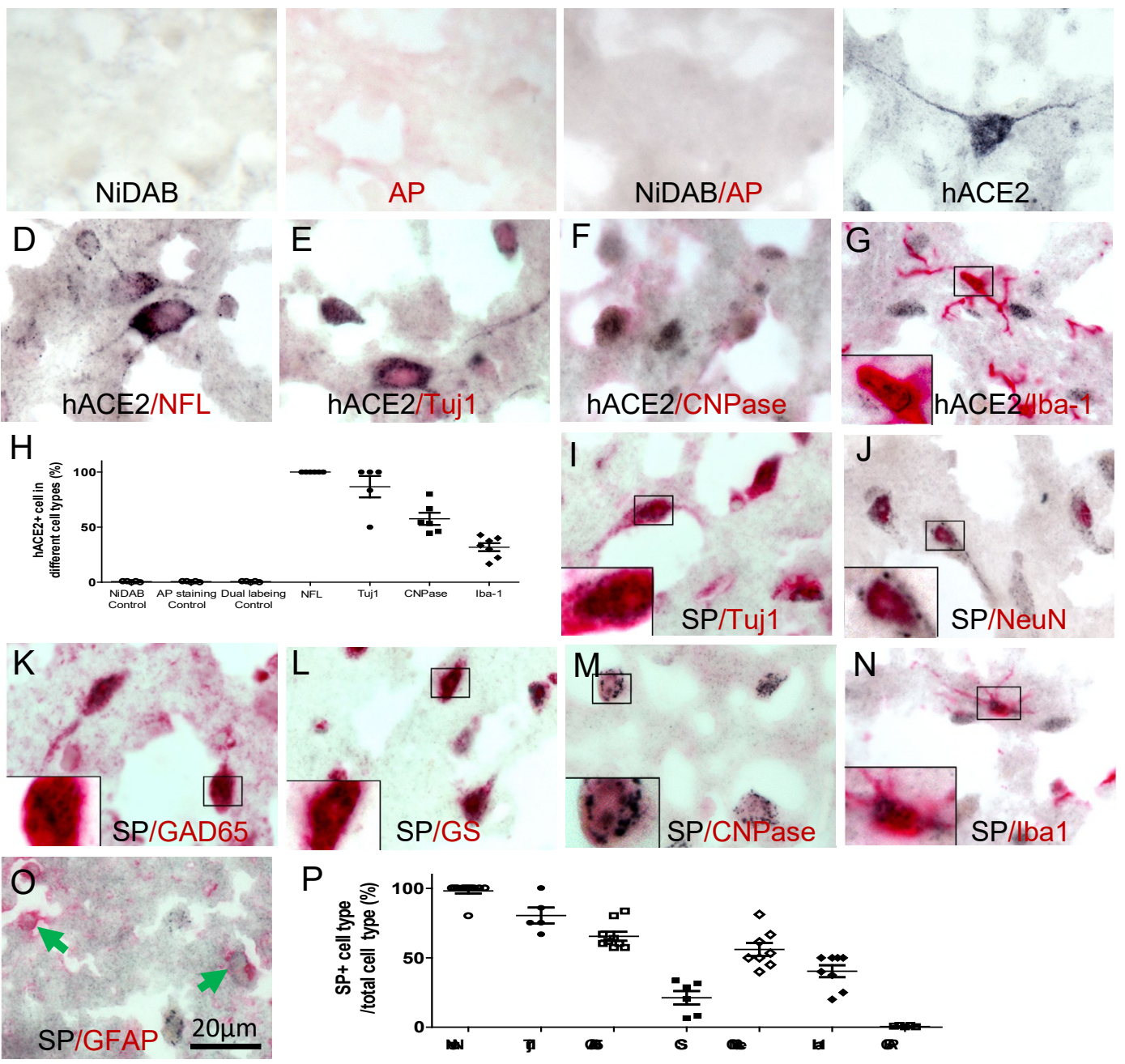


Fig. 3

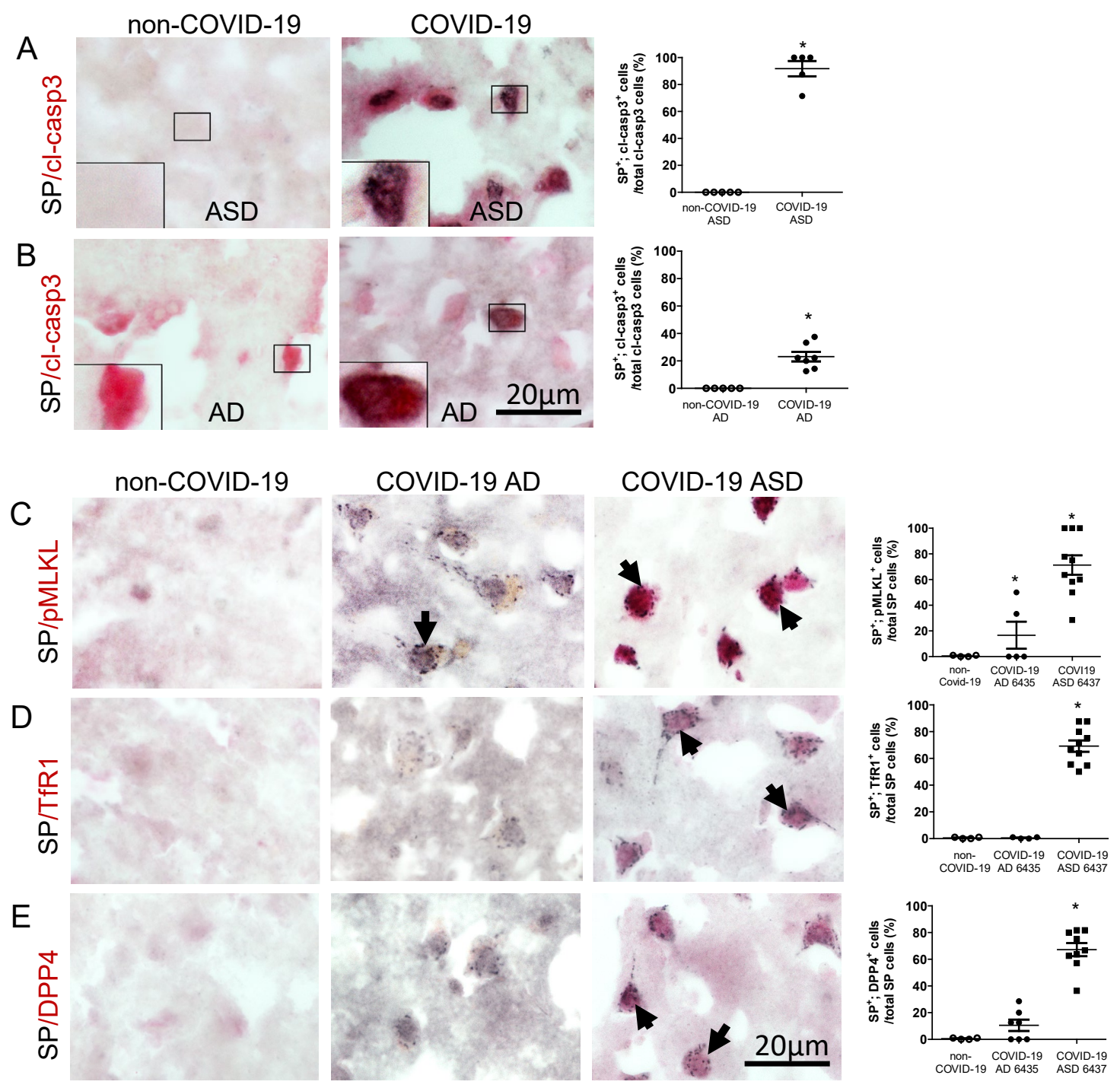


Fig. 4

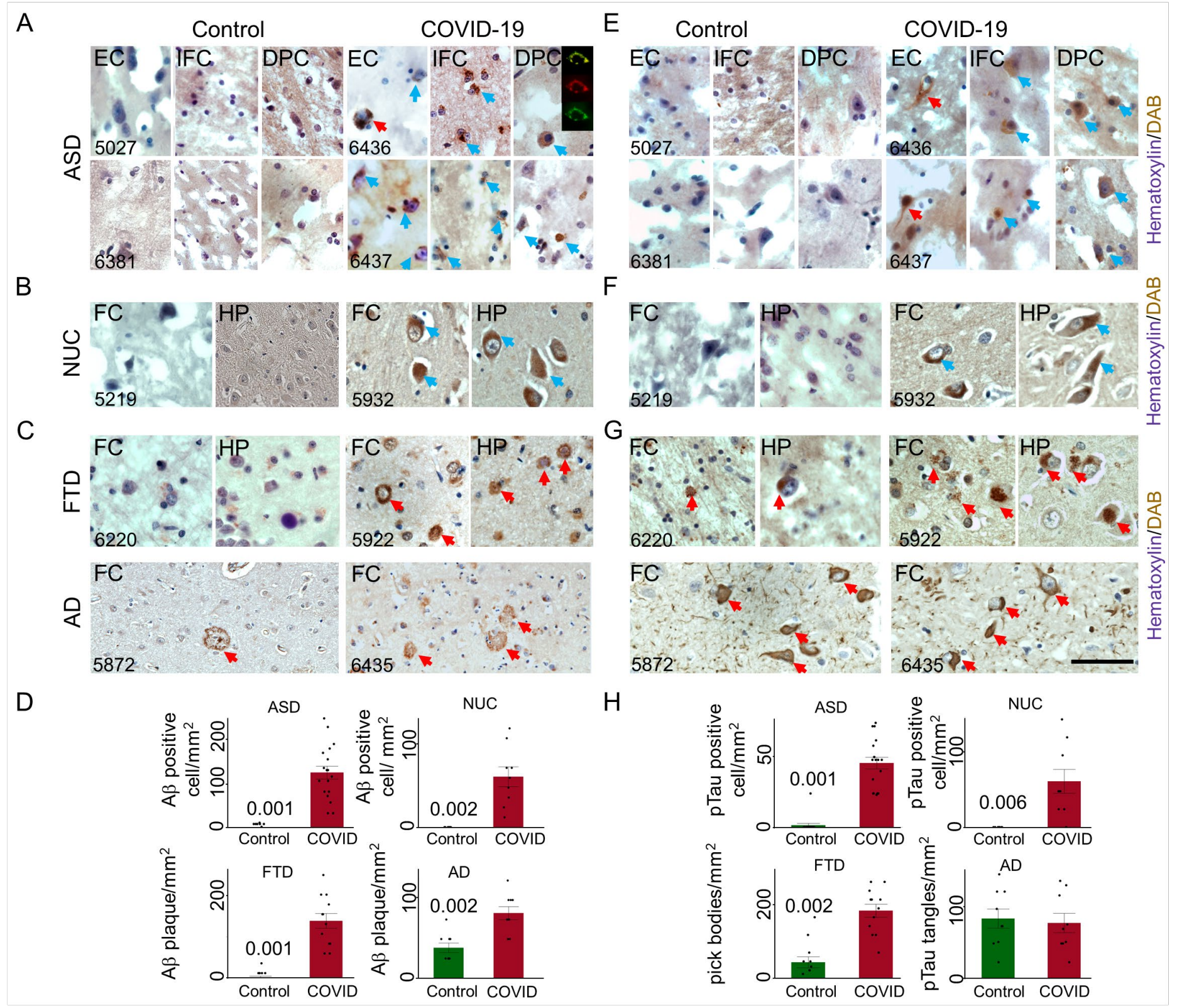


Fig. 5

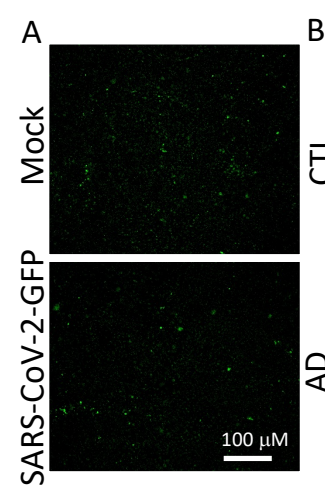

B
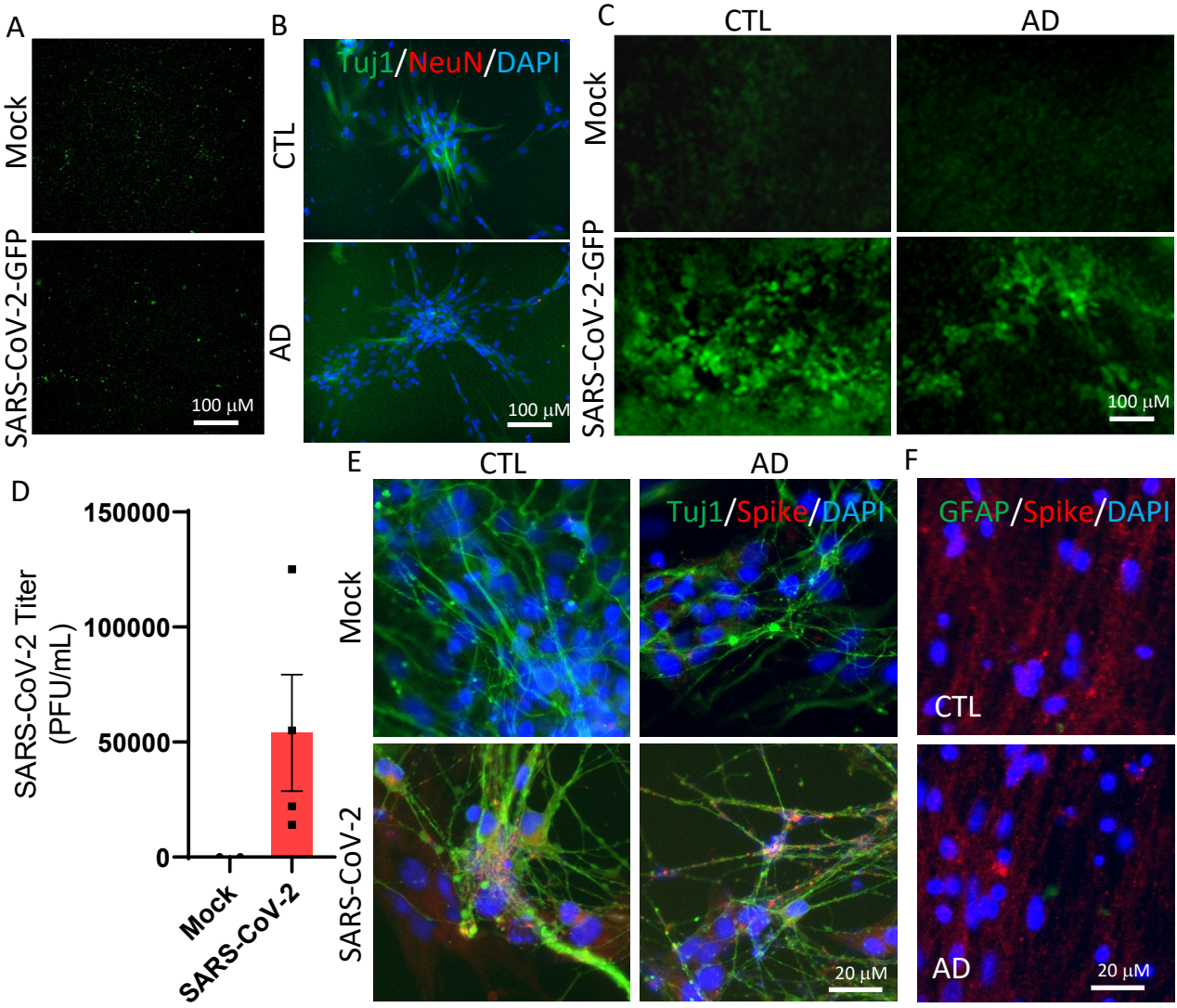

AD F

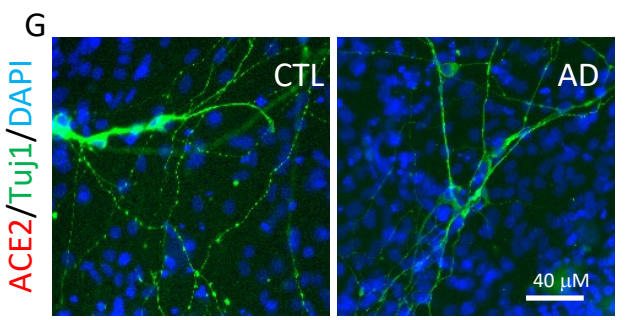

$\mathrm{H}$
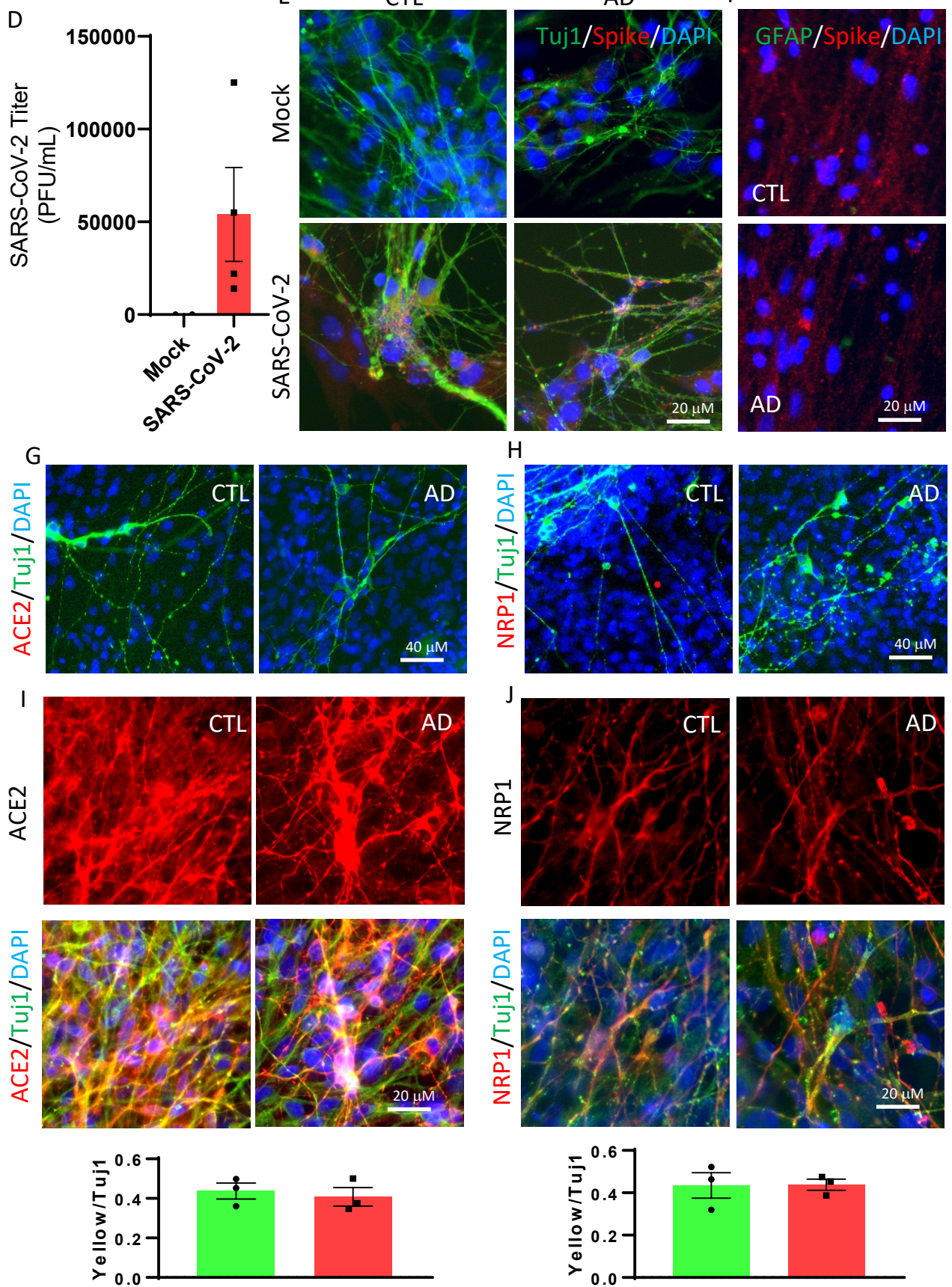


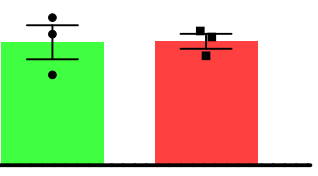
Fig. 6
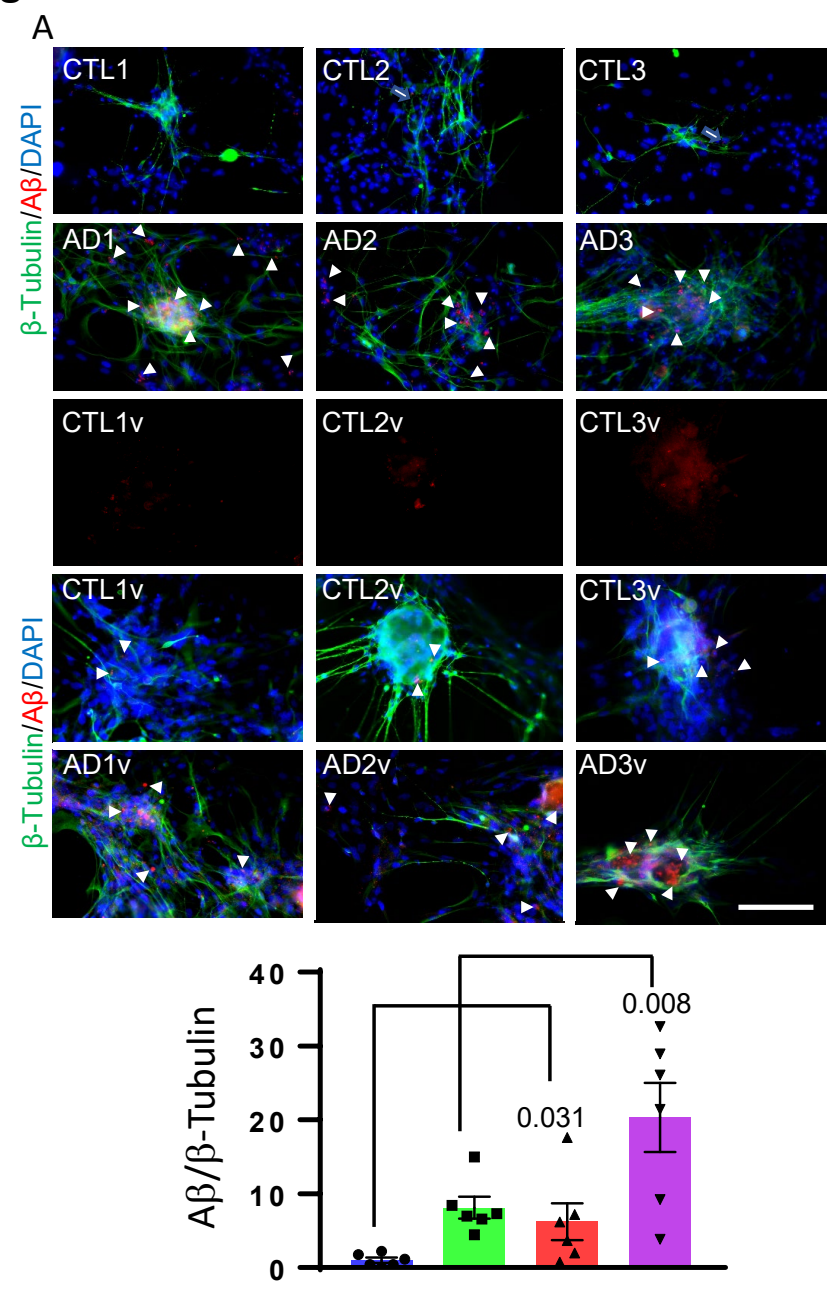

$$
\text { D }
$$

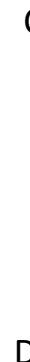

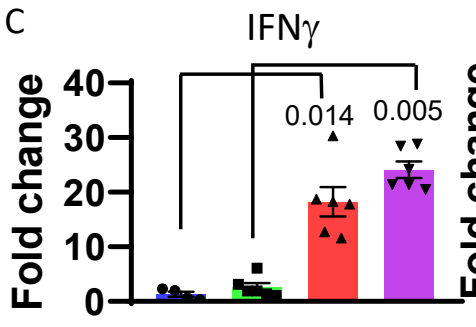
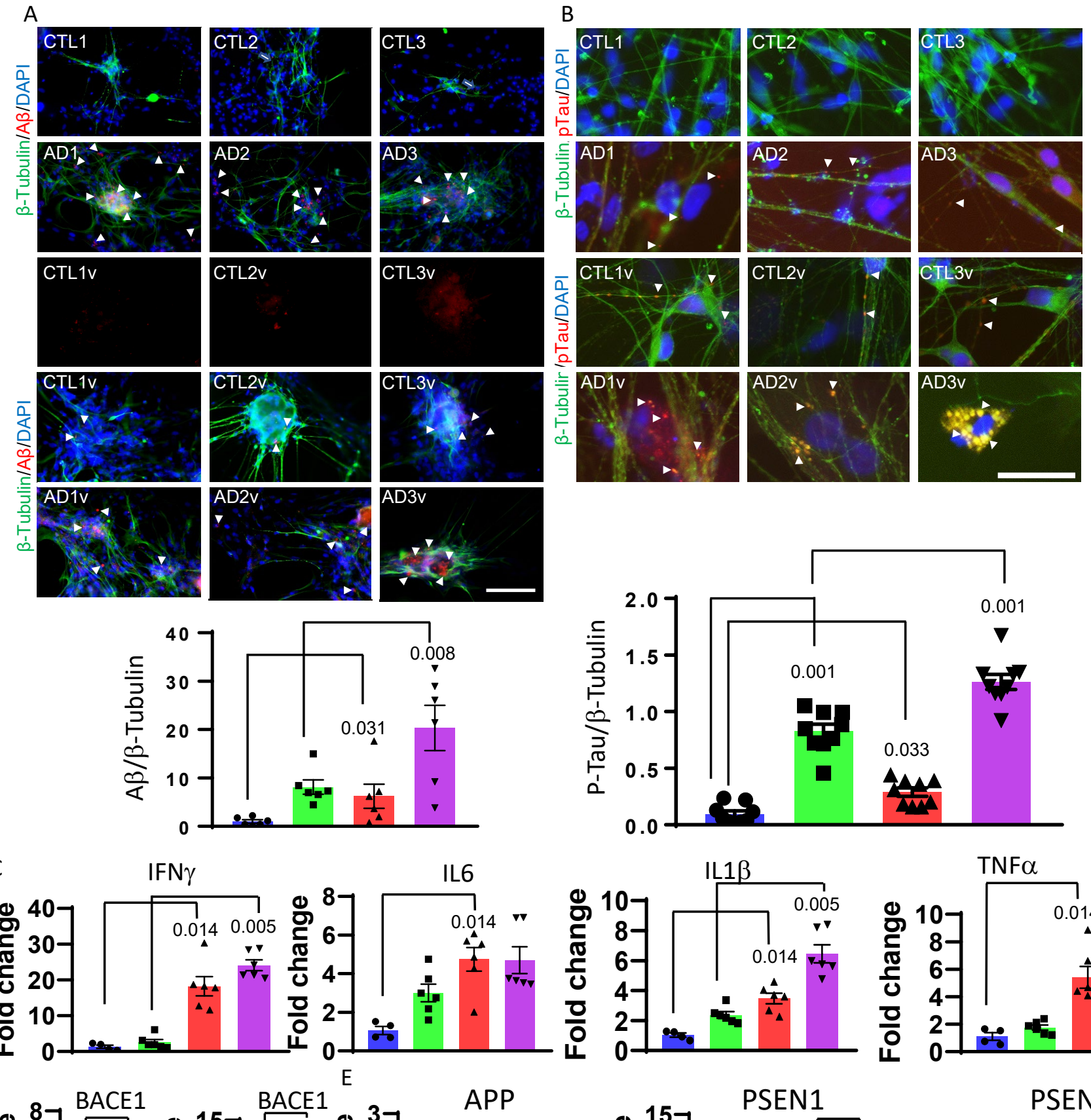

8ᄀ BACE1 BACE1 ${ }^{E}$ APP

12

은

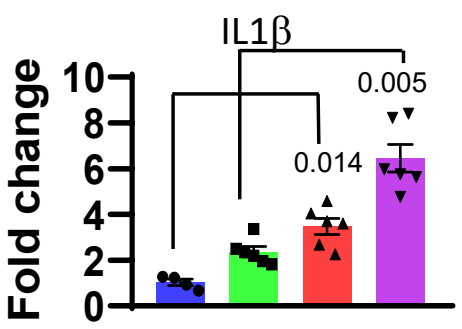

PSEN1

PSEN2

Fig. 7

A

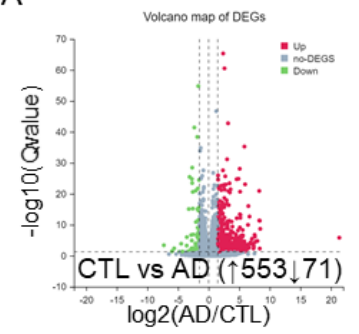

B

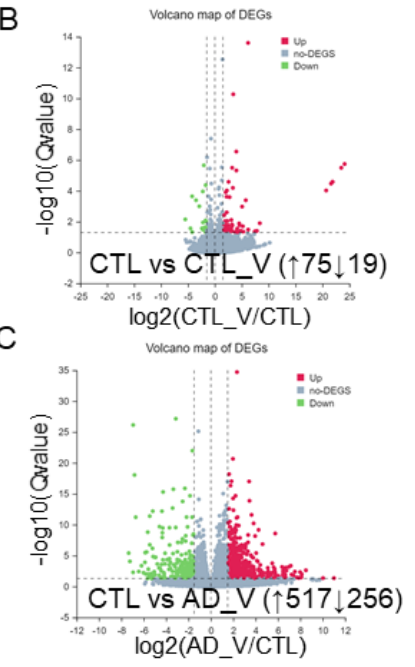

F

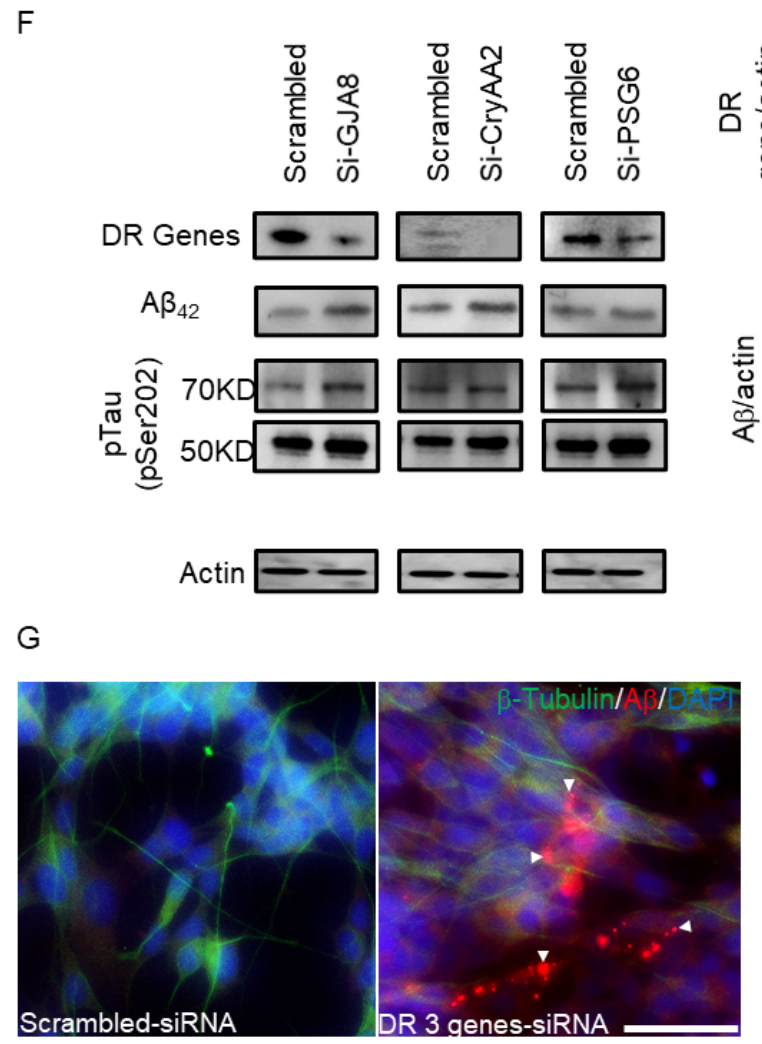

G
D

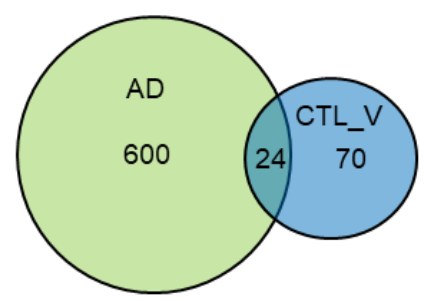

E

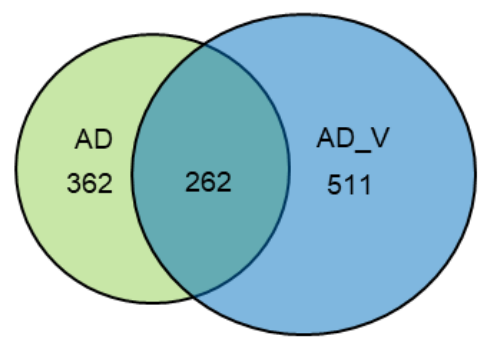

KEGG Pathway Term Level2

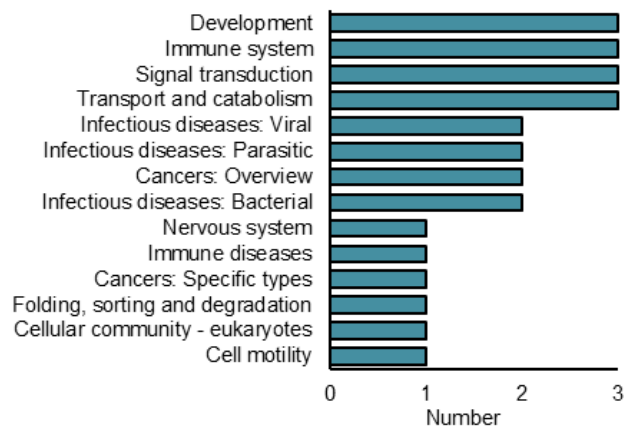

KEGG Pathway Term Level2 (top 20)

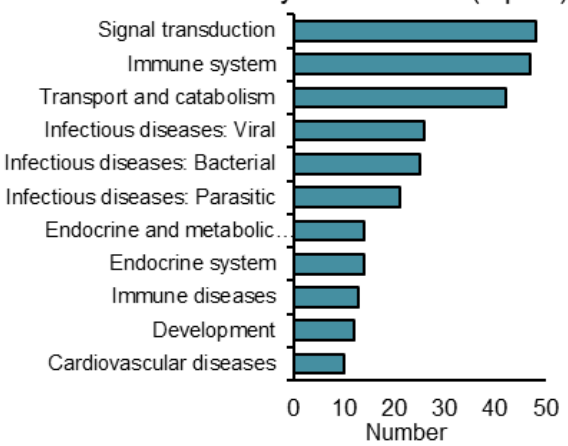

Infectious diseases: Bacterial

Infectious diseases: Parasitic

Endocrine and metabolic

ndocrine system

Development
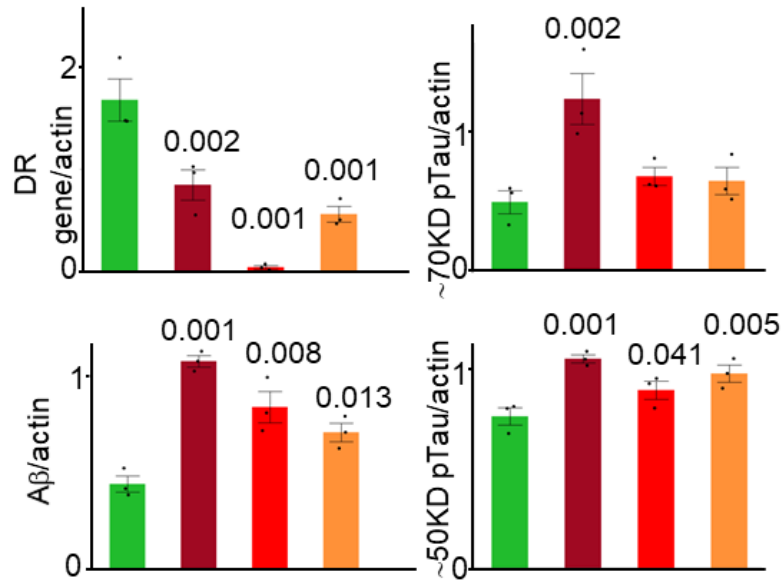

- Scrambled

- Si-GJA8
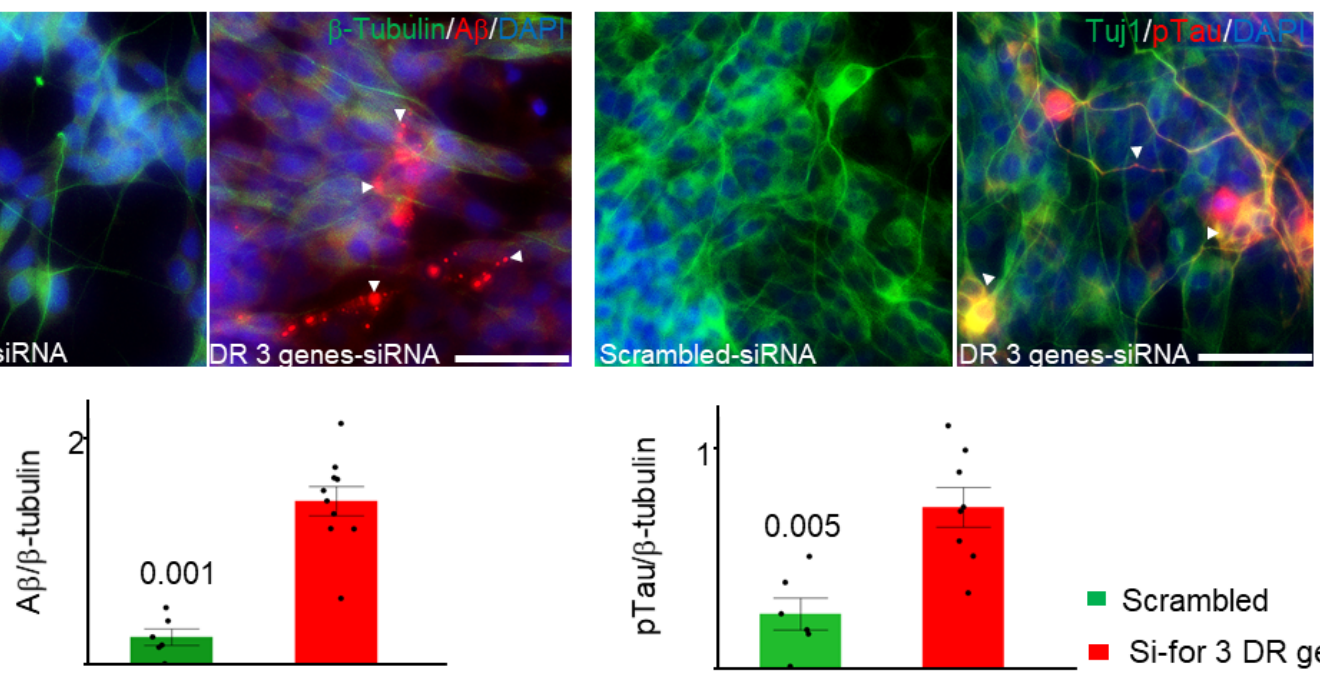

- Scrambled

- Si-for 3 DR genes 


\section{Methods}

\section{Mammalian iPSCs and culture conditions}

Human iPSCs ( 1 cases of familial AD, 2 cases of sporadic AD, and 3 cases of apparently healthy controls) purchased from the Coriell Institute for Medical Research were used in our study. iPSCs were maintained on irradiated mouse CF-1 feeder layer (ATCC, Manassas, VA) at $37{ }^{\circ} \mathrm{C}$ and $5 \%$ $\mathrm{CO}_{2}$ in Knockout DMEM medium (Invitrogen, Waltham, MA) supplemented with $20 \%$ Knockout Serum Replacer (KSR) (Invitrogen, Waltham, MA), $0.1 \mathrm{mM}$ nonessential amino acids (Invitrogen, Waltham, MA), 2 mM GlutaMAX (Invitrogen, Waltham, MA), 0.1 mM $\beta$-Mercaptoethanol (Sigma-Aldrich, St. Louis, MO), $10 \mathrm{ng} / \mathrm{ml}$ recombinant human basic fibroblast growth factor (Invitrogen, Waltham, MA) (hiPSC medium).

\section{Viral strains}

Vero E6 cells were maintained in EMEM (ATCC) media with 10\% Serum Plus II Medium Supplement (Sigma-Aldrich). SARS-CoV-2 virus were obtained from the CDC following isolation from a patient in Washington State (WA-1 strain - BEI \#NR-52281). SARS-CoV-2 GFP was generously provided by Dr. Ralph S. Baric. Stocks were prepared by infection of Vero E6 cells.

\section{Brain Tissues}

The major source for postmortem tissues is the Brain and Tissue Bank at University of Maryland (UMB). The Brain and Tissue Bank is a brain and tissue repository of the NIH NeuroBioBank. The Brain Tissue Bank is a national resource for investigators utilizing human post-mortem brain tissues and related biospecimens for research in understanding the conditions of the nervous system. All brain tissue is procured, stored, and distributed according to applicable State and 
Federal guidelines and regulations involving consent, protection of human subjects and donor anonymity. All brain tissues we obtained from the Brain Tissue Bank are de-identified.

Following formalin-fixed brain tissues were obtained from the Brain and Tissue Bank at UMB: COVID-19 autism (ASD, ID \#6436, 38 year-old, \#6437, 30 year), COVID-19 Alzheimer's disease (AD, \#6535, 77 year), age-matched non-COVID-19 controls (6 cases), non-COVID-19 ASD (6 cases), non-COVID-19 FTD (frontotemporal dementia, 4 cases), and apparently healthy subjects (9 cases). We also obtained 4 cases of non-COVID-19 AD from the NIH NeuroBioBank. Samples of three brain regions were obtained from individual: dorsolateral prefrontal cortex (Broca area 8), inferior frontal cortex (Broca area 44), entorhinal cortex.

Following frozen brain tissues were also obtained from the Brain and Tissue Bank. The frozen tissues were used for RNA extraction followed by reverse transcription and PCR detection of SARS-CoV-2 with CDC specific primers. The frozen tissues include SARS-CoV-2 infected lung and brain tissues (ASD, \#6436), age-matched non-COVID-19 ASD controls and FTD controls. In addition, paraffin sections and frozen brain tissues of the subjects of COVID-19 FTD (1 case) and COVID-19 individual without underlying condition, and non-COVID-19 AD (3 cases) were obtained from the Biomarker Laboratory and Biorepository at University of Southern California Alzheimer's Therapeutic Research Institute (USC ATRI) at University of California San Diego (UCSD).

\section{Neuronal Cells}

Primary Human Neurons were obtained from Neuromics (CA3 Bioscience, MN, USA). The cells were maintained in neuron growth medium supplemented (CA3 Bioscience, MN, USA) with 10\% FBS at $37{ }^{\circ} \mathrm{C}$ in a humidified atmosphere of $5 \% \mathrm{CO}_{2}$ in culture flask. 


\section{Immunohistology staining of spike protein and markers of neural cells and programmed cell}

\section{death}

The formalin-fixed brain tissue blocks were immersed in $30 \%$ sucrose, then embed in OCT cryostat sectioning medium. The tissues were sectioned on a cryostat at $10 \mu \mathrm{m}$. After rehydrated, the sections were treated with $2 \% \mathrm{H}_{2} \mathrm{O}_{2}$ for 15 minutes to quench the endogenous peroxidase. The staining was performed as previously described $[1,2]$. Briefly, the slices were incubated with blocking solution consisting of 4\% normal donkey serum (NDS), 0.2\% triton (TX)-100 in phosphate-buffered saline (PBS) for $60 \mathrm{~min}$, then with primary antibody diluted in blocking solution at $4{ }^{\circ} \mathrm{C}$ overnight. The slices were washed with PBS and incubated for $1 \mathrm{~h}$ in a biotinylated secondary antibody anti-mouse or rabbit (depending on the host species producing the primary antibodies). After three times of PBS washes, the sections were incubated in ABC solution (1:500; Vectastain Elite Kit) for $1 \mathrm{~h}$. The $\mathrm{ABC}$ solution was prepared and placed on ice for 30 minutes before use. Antibody labeling was then visualized via precipitation with a diaminobenzidine (DAB, $10 \mathrm{mg} / 50 \mathrm{ml}$ ) chromogen solution in PBS $+0.003 \% \mathrm{H}_{2} \mathrm{O}_{2}$ (brown products) or with DAB in $0.175 \mathrm{M}$ sodium acetate $+0.5 \%$ nickel ammonium sulfate $+0.003 \% \mathrm{H}_{2} \mathrm{O}_{2}$ (for $\mathrm{Ni}-\mathrm{DAB}$ staining, black products). If necessary, the sections were counterstained with hematoxylin before coverslip.

For immunohistology dual labeling, the sections were subject Ni-DAB staining with first primary antibody as described above. After PBS wash, the sections were re-blocked for 1 hours and following incubation with the second primary antibody at $4^{\circ} \mathrm{C}$ overnight. Next day, the sections were washed with PBS and incubated with alkaline phosphatase (AP)-conjugated goat secondary antibody (1:200) for $1 \mathrm{~h}$, followed by incubation with AP red substrate (1:100) for 20-30 minutes. DAB-stained sections were counterstained with hematoxylin. Sections were immersed in hematoxylin solution for $3 \mathrm{~min}$ and rinsed in running tap water until rinse water is colorless. After 
differentiation by dipping slides 10 times in acid rinse solution $(1 \%$ hydrochloric acid $(\mathrm{HCl})$ in $70 \%$ ethanol), the slides were incubated in the bluing solution (1.5\% lithium carbonate) for $30 \mathrm{~s}$. There is no hematoxylin counterstaining performed for Ni-DAB or Ni-DAB/AP dual labeling before dehydrate and coverslip.

Dehydrate and coverslip sequence: 70\% ethanol, 3 minutes; 95\% ethanol I and II, 3 minutes for each; 100\% ethanol I and II, 3 minutes for each; xylene I and II, 5 minutes for each. Mounting medium: Permount Mounting Medium.

\section{$A \beta$ and $p-$ Tau Immunohistochemistry}

$\beta$-amyloid $(\mathrm{A} \beta)$ and phospho-tau (pTau) Immunohistochemistry was carried out as previously described [3]. Briefly, the coronal sections were rehydrated, and endogenous peroxidase was quenched by treating with $0.3 \% \mathrm{H}_{2} \mathrm{O}_{2}$. For $\mathrm{A} \beta$ staining, the antigen retrieval was carried by using $70 \%$ formic acid for 20 minutes. Antigen retrieval for the other antibodies was by autoclaving at $120^{\circ} \mathrm{C}$ for $5 \mathrm{~min}$. The primary antibody was applied on the sections at $4^{\circ} \mathrm{C}$ overnight, followed by 60-min incubation with specific secondary antibody coupled with HRP (Histofine simple stain MaxPo M/R, Nichirei Bioscience Inc., Japan) at room temperature. DAB reaction was performed to visualize the color. All antibody dilutions and washing steps were performed in phosphate buffer, pH 7.2. HRP intensities and cell counts in 3 different regions (Frontal Cortex, Entorhinal Cortex) in three sections/slices from each group were measured using the ImageJ Fiji platform. Data were represented as staining intensity per square millimeter. Because the MaxPo M/R antibodies are Fab fragments, the blocking is not necessary.

\section{Thioflavin staining}

The A $\beta$ staining Thioflavin-T were performed following the similar antigen protocol. After blocking with $5 \%$ goat serum for 30 minutes overnight incubation with anti A $\beta$ antibody $6 \mathrm{E} 10$ 
were performed. In the following day the brain sections were probed with Alexa-fluor594 labeled secondary antibody. After an extensive washing, Thioflavin-T (0.1\% in 50\% DMSO) were applied over the brain sections for 5 minutes. An extensive washing was performed using PBS containing 0.05\% tween20. Furthermore, the fluorescent background was quenched with Tureblack (Biotium, USA). Thioflavin-T and Alexa-fluor594 intensity were measured using a fluorescence laser microscope (LSM780, Zeiss, Germany).

\section{Immunofluorescence staining}

For immunofluorescence staining, cells were cultured on a collagen-coated coverslip. After specific treatments, cells were fixed with $4 \%$ paraformaldehyde-PBS. The cell membrane was permeabilized with $0.25 \%$ Triton-X100 and/or $5 \mathrm{mg} / \mathrm{ml}$ digitonin followed by blocking with $5 \%$ BSA. After probing with primary antibodies, specific Alexa-fluor labeled secondary antibody were used. 4',6-Diamidino-2-phenylindole dihydrochloride (DAPI) staining was used to visualize the nuclei (Thermo Fisher Scientific). Fluorescence was assessed using a fluorescence laser microscope (LSM780, Zeiss, Germany).

\section{Detection of SARS-CoV-2 RNA using RNAscope}

RNA In situ hybridization in FFPE slides was performed by using the RNAScope 2.5 HD Detection (RED) Kit (ACD, CA), based on the protocol provided by the manufacturer. Briefly, after deparaffinization and antigen retrieval, the slides were hybridized with the $40-\mathrm{ZZ}$ positivesense RNA probe, V-SARS-CoV-2-S (Ref\#: 854841, ACD) in the oven at $40{ }^{\circ} \mathrm{C}$ for 2 hours. We then washed the slides with $1 \mathrm{X}$ Wash Buffer for 2 min twice. The remaining hybridization procedure at $40{ }^{\circ} \mathrm{C}$ include: 1) Amp 1, $30 \mathrm{~min}$; 2) Amp 2, 15 min; 3) Amp, $30 \mathrm{~min}$; 4) Amp 4, 15 min; 5) Amp 5, 30 min, and 6) Amp 6, 15 min with an interval of repeated 1X Wash buffer for 2 min. Subsequently, the signals were detected by incubating with a mixture of RED-B and RED-A 
at ratio of 1:60 for $10 \mathrm{~min}$ at room temperature followed by counterstaining with $50 \%$ hematoxylin for $2 \mathrm{~min}$.

\section{Generation of human induced pluripotent stem cells (hiPSCs)}

Human fibroblast from Coriell Institute were induced into pluripotent stem cell with CMV promoter Thomson factors lentivirus set which contains Lenti-virus harboring Oct4, Sox2, Nanog, and Lin28 (Cat\#: G353, ABM Inc., Canada). Briefly, human fibroblasts were seeded on 6 well plate with $8 \times 10^{4}$ per well at day -2 and transduced with Lenti-virus particles (MOI of 4:3:3:3). Cells were cultured for 7 days and replated at $2 \times 10^{4}$ cells per well of 6 well plate on irradiated mouse embryonic fibroblasts (MEF) feeder layer. From day 8, medium was replaced with Knockout DMEM (Invitrogen) supplemented with 20\% Knockout Serum Replacer (KSR, Invitrogen), 0.1 mM nonessential amino acids (Invitrogen), $2 \mathrm{mM}$ GlutaMAX (Invitrogen), $0.1 \mathrm{mM} \beta$-mercaptoethanol (Sigma-Aldrich, St. Louis, MO), $10 \mathrm{ng} / \mathrm{ml}$ recombinant human basic fibroblast growth factor (Invitrogen) (hiPSC medium). On week 3-4, iPSCs were identified from morphology change and picked out into 48-well plates with MEF feeder.

\section{Neuron differentiation from hiPSCs.}

hiPSCs were differentiated into neurons as described with modification (Nat Med. 2018 May;24(5):647-657). hiPSCs dissociated with collagenase IV (Stem Cell Technologies) were cultured in suspension to form embryoid bodies (EB) in hES medium without bFGF for 5 days followed by maintenance in NIM medium containing Dulbecco's modified Eagle's medium/F12 and Neurobasal Medium (1:1, Thermo Fisher), 1\% N2 Supplement (Life Technologies), 1\% B27 Supplement (Life Technologies), nonessential amino acids, and 0.5\% penicillin/streptomycin (Life Technologies) supplemented with inhibitors of the TGF- $\beta$ receptor (SB431542, Stemgent; $5 \mu \mathrm{M})$ and the bone morphogenetic protein receptor (LDN-193189, Stemgent; $0.25 \mu \mathrm{M})$. On day 7 , 
spheres were transferred to wells coated with Matrigel (BD Biosciences) and grown in NPM medium which is same to NIM medium but replace SB431542 and LDN-193189 with $10 \mathrm{ng} / \mathrm{ml}$ bFGF (PeproTech), $10 \mathrm{ng} / \mathrm{mL}$ epidermal growth factor (EGF) (PeproTech), and $2 \mu \mathrm{g} / \mathrm{ml}$ heparin (Sigma). On day 15, medium was changed to NDM medium which is same to NPM medium but replace bFGF, EGF and heparin in NPM with brain-derived neurotrophic factor (10 ng/ml; PeproTech), and glial cell-derived growth factor (10 ng/ml; PeproTech). Around day 20, neurons were observed and were further differentiated for 30 days.

\section{Immunofluorescence staining in iPSCs-derived neurons}

Neurons derived from AD or Control (from healthy individuals) hiPSCs with or without SARS-CoV-2 infection were fixed in 4\% paraformaldehyde (PFA) for 10 minutes followed by blocking in 5\% bovine serum albumin in PBST (0.1\% Triton X-100 in PBS) for 10 minutes. The following antibodies were used as primary antibodies: Spike (1:200), Tuj1 (1:500), ACE2 (1:200), NRP1 (1:200), NeuN (1:200), GFAP (1:200), c-Cas3 (1:200). Normal rabbit or mouse IgG using the same dilutions as primary antibodies were used as controls. After washing with PBS, neurons were incubated with secondary antibodies. Then, neurons were counterstained with DAPI and mounted with aqueous mounting medium (Sigma, St Louis, MO). Images were captured under a microscope (Keyence BZ X700, Osaka, Japan).

\section{SARS-CoV-2 production and infection of hiPSCs derived neurons}

The stock of SARS-CoV-2 virus (CDC, WA-1 strain - BEI \#NR-52281, and SARS-CoV-2 GFP generously provided by Dr. Ralph S. Baric, at the Department of Epidemiology and Department of Microbiology and Immunology, University of North Carolina at Chapel Hill), were prepared by infection of Vero E6 cells for two days when CPE (cytopathic effects) was starting to become visible. Media were collected and clarified by centrifugation prior to being aliquoted for storage 
at $-80{ }^{\circ} \mathrm{C}$. Neurons derived from hiPSCs were infected with SARS-CoV-2 at $5 \times 10^{3}, 1 \times 10^{4}$, or $2 \times 10^{4}$ plaque forming units (pfu) per well of 6 well plate for $48 \mathrm{~h}$ in NDM medium. All work with infectious virus was performed in a Biosafety Level 3 laboratory and approved by our Institutional Biosafety Committee.

\section{SARS-CoV-2 Titering by semi-solid plaque assay}

VeroE6 cells were plated in 12 well plates with $2 \times 10^{5}$ cells per well one day prior to processing. On the day of processing, samples were serially diluted 1:10 and 200uL of each sample dilution was added to each well in singlet and incubated for 1 hour at $37^{\circ} \mathrm{C}(5 \% \mathrm{CO} 2)$ with rocking every 15 minutes. Following incubation, $2 \mathrm{~mL}$ of a semi-solid agar overlay, DMEM (gibco) containing 4\% fetal bovine sera (gibco) and 2\% agarose, was added to each well. Plates are incubated for 3 days at $37^{\circ} \mathrm{C}(5 \% \mathrm{CO} 2)$ before plates were fixed with $4 \%$ paraformaldehyde, stained with crystal violet stain, and plaques counted.

\section{Neural maturation, siRNA Transfection, and Lenti virus transduction}

Primary Human Neurons obtained from Neuromics (Edina, MN) grow for four weeks in the Neuromics growth medium supplemented with brain derived neurotropic factor (BDNF). Medium were changed every 2 days. For further experiment, cells were plated on a 6-well plate in neuron growth medium supplemented with 5\% FBS. siRNA transfection was carried out using XtremeGENE siRNA transfection reagent (Roche, Basel, Switzerland) at $60 \%$ cell confluency. Medium was replaced with fresh medium 24 hours post-transfection and maintained for additional 48 hours. Lentivirus mediated overexpression of the FCGR, LILRB5 and OTOR carried out using virus particles obtained from OriGene (Rockville, MD). Virus transduction were carried out at 5.0 MOI using polybrene. GFP expressing lenti-particles were used as the control. 


\section{RNA sequencing}

mRNAs were extracted from neurons derived from AD or Control hiPSCs with or without SARSCoV-2 infection and sequenced at BGI on DNBSeq platform. Briefly, mRNAs were extracted with Trizol reagent. First-strand cDNA was generated using random hexamer-primed reverse transcription, followed by a second-strand cDNA synthesis. The synthesized cDNA was subjected to end-repair and then was 3' adenylated. Adapters were ligated to the ends of these 3' adenylated cDNA fragments, followed by PCR. PCR products were purified with Ampure XP Beads and dissolved in EB solution. Library was validated on the Agilent Technologies 2100 bioanalyzer. The double stranded PCR products were heat denatured and circularized by the splint oligo sequence. The single strand circle DNA were formatted as the final library. The library was amplified with phi29 to make DNA nanoball (DNB) which had more than 300 copies of one molecular. The DNBs were load into the patterned nanoarray and single end 50 (pair end 100) bases reads were generated in the way of sequenced by synthesis.

\section{Quantitative reverse transcription PCR}

mRNAs were extracted from neurons derived from AD or Control hiPSCs with or without SARSCoV-2 infection in Trizol reagent (Thermofisher, Waltham, MA) and followed by cDNA synthesis with SuperScript ${ }^{\mathrm{TM}}$ III Reverse Transcriptase kit (ThermoFisher). RT-PCR were performed with PowerUp ${ }^{\text {TM }}$ SYBR ${ }^{\text {TM }}$ Green Master Mix (ThermoFisher) with 45 cycles and PCR products were loaded into $2 \%$ agarose gel and run at $75 \mathrm{v}$ for $5.5 \mathrm{~h}$. Gel Image were recorded with Bio-Rad ChemiDoc MP imaging system (Hercules, CA).

\section{QUANTIFICATION AND STATISTICAL ANALYSIS}

In the experiment assessing the impact of SARS-CoV-2 virus on the brain pathology, we analyzed

(a) 5 Covid19 samples consisting of AD-Covid19 (1 case), FTD-Covid19 (1 case), ASD-Covid19 
( 2 cases), and Covid-19 without underlying condition ( 1 case); (b) 6 cases of sporadic AD as the controls; and (c) 8 cases of age-matched, non-Covid-19 healthy controls. Labeled cells from at least 3 random fields $(n \geq 3)$ were counted. Data are presented as the means \pm standard errors (SEs). Student's t test was used for two group comparisons. One-way ANOVA was performed for comparisons of more than two group using graphPad prism software v7. In ANOVA, a Tukey test was used to estimate the significance between groups. Differences were considered statistically significant when $P<0.05$.

In the experiment with cell culture studies, we differentiated neurons from 3 iPSC lines derived from AD fibroblast cells and 3 lines from healthy subjects as the controls. The experiments were repeated in triplicate as we previously described $[4,5]$. Data are presented as the means \pm standard errors (SEs). Student's $t$ test was used for two group comparisons. One-way ANOVA plus Tukey test was performed for comparisons of more than two group. Differences were considered statistically significant when $P<0.05$.

\section{Reference:}

[1] Shen WB, McDowell KA, Siebert AA, Clark SM, Dugger NV, Valentino KM, et al. Environmental neurotoxin-induced progressive model of parkinsonism in rats. Ann Neurol. 2010;68:70-80.

[2] Shen WB, Plachez C, Tsymbalyuk O, Tsymbalyuk N, Xu S, Smith AM, et al. Cell-Based Therapy in TBI: Magnetic Retention of Neural Stem Cells In Vivo. Cell Transplant. 2016;25:1085-99.

[3] Elahi M, Hasan Z, Motoi Y, Matsumoto SE, Ishiguro K, Hattori N. Region-Specific Vulnerability to Oxidative Stress, Neuroinflammation, and Tau Hyperphosphorylation in Experimental Diabetes Mellitus Mice. J Alzheimers Dis. 2016;51:1209-24.

[4] Xu C, Shen W-B, Reece EA, Hasuwa H, Harman C, Kaushal S, et al. Maternal diabetes induces senescence and neural tube defects sensitive to the senomorphic rapamycin. Science Advances. 2021;7:eabf5089.

[5] Wang F, Wu Y, Gu H, Reece EA, Fang S, Gabbay-Benziv R, et al. Ask1 gene deletion blocks maternal diabetes-induced endoplasmic reticulum stress in the developing embryo by disrupting the unfolded protein response signalosome. Diabetes. 2015;64:973-88. 
Table S1. Relative numbers of spike protein ${ }^{+}$cells in 5 COVID-19 cases

\begin{tabular}{llcccc}
\hline ID\# & Disease & $\underline{\text { Age }}$ & $\underline{\text { EC }}$ & $\underline{\text { DPC }}$ & $\underline{\text { IFC }}$ \\
$\mathbf{6 4 3 5}$ & COVID-19, AD & 77 & ++ & ++ & ++ \\
$\mathbf{6 4 3 6}$ & COVID-19, ASD & 38 & ++ & ++ & ++ \\
$\mathbf{6 4 3 7}$ & COVID-19, ASD & 30 & ++ & ++ & +++ \\
$\mathbf{5 9 3 2}$ & COVID-19, NUC & 71 & Not tested & Not tested & + \\
$\mathbf{5 9 2 2}$ & COVID-19, FTD & 70 & Not tested & Not tested & + \\
\hline
\end{tabular}

AD: Alzheimer's disease; ASD: autism; EC: entorhinal cortex; DPC: dorsolateral prefrontal cortex; IFC: inferior frontal cortex. FTD: frontotemporal dementia. NUC: no underlying condition. +++: $>200$ spike protein ${ }^{+}$cells $/ \mathrm{mm}^{2} ;++$ : 100-200 spike protein ${ }^{+}$cells $/ \mathrm{mm}^{2} ; \quad+:<100$ spike protein ${ }^{+}$cells $/ \mathrm{mm}^{2}$ 
Fig. S1
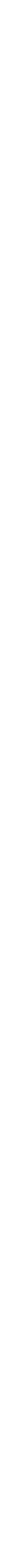

Covid-19 ASD
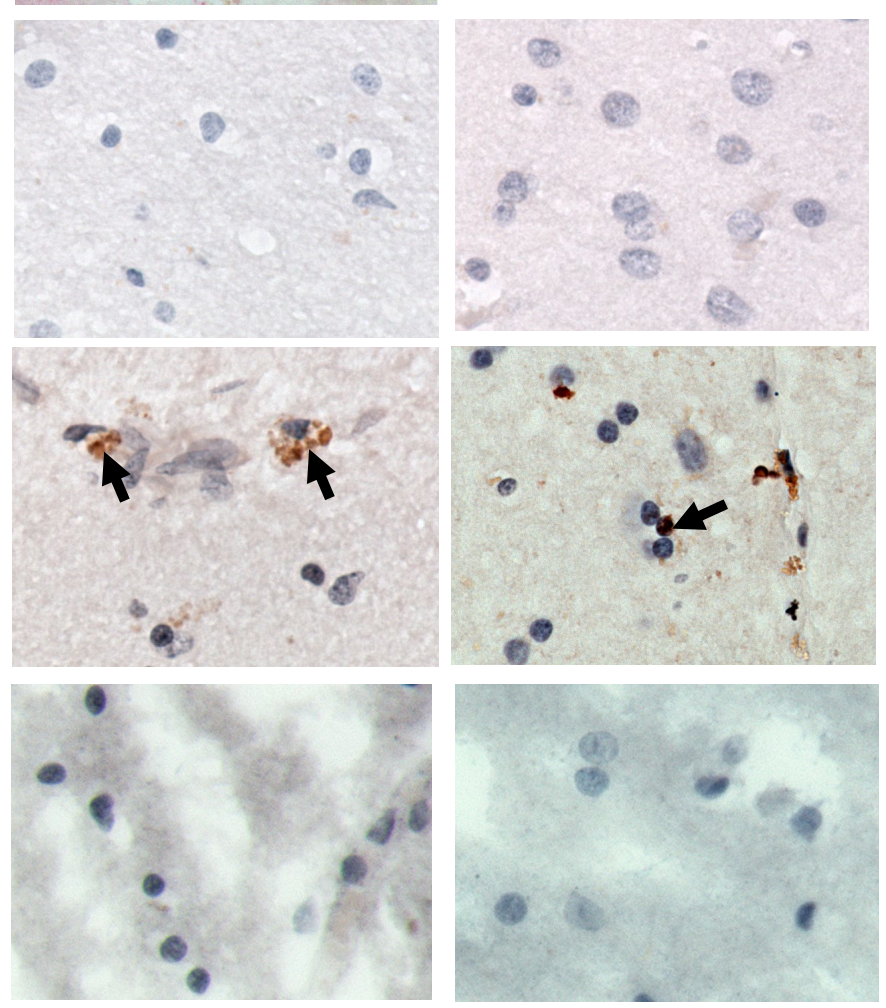
Fig. S2

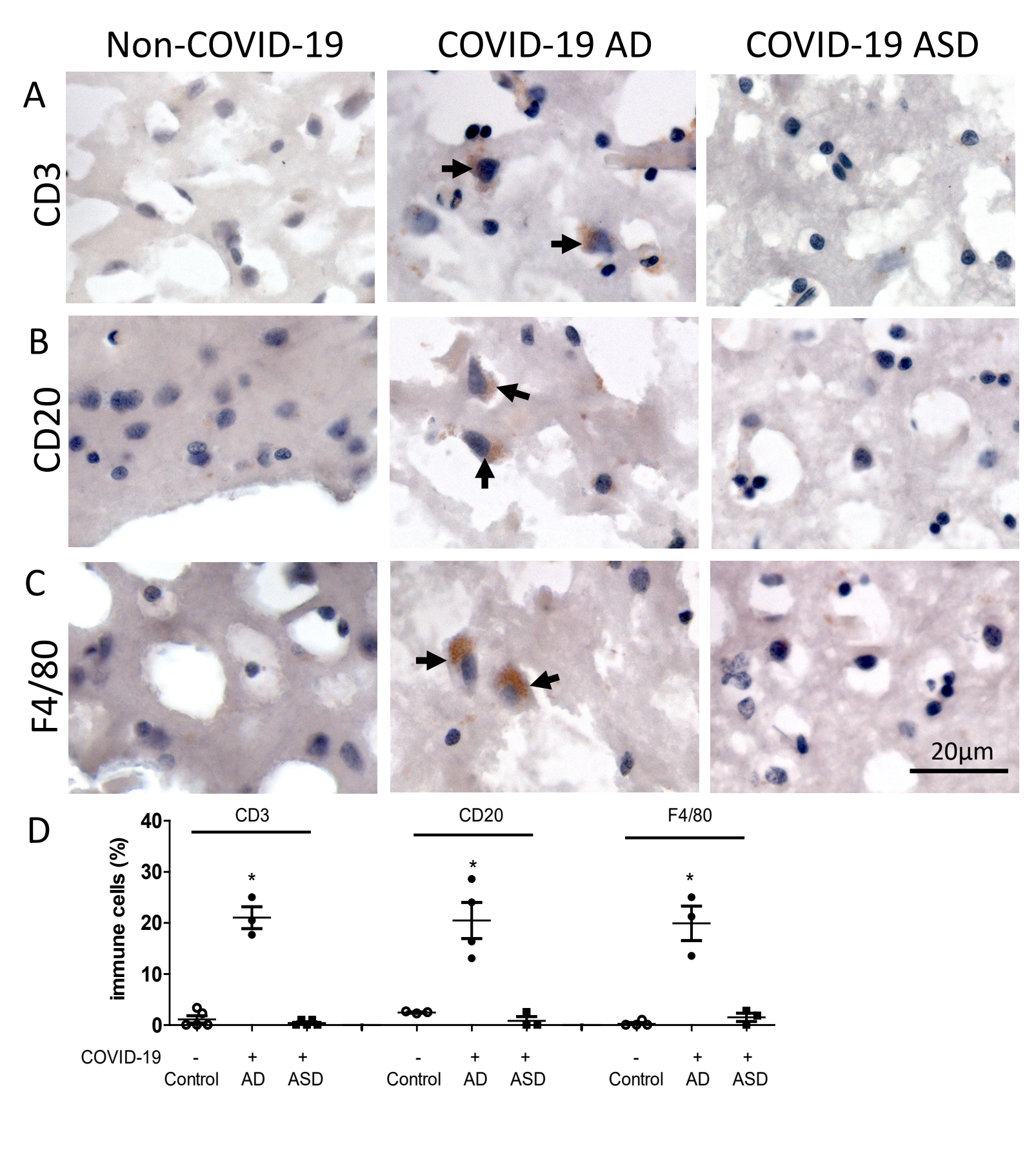


Fig. S3

A

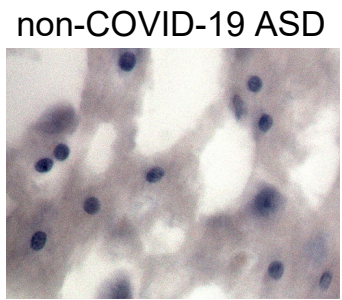

B

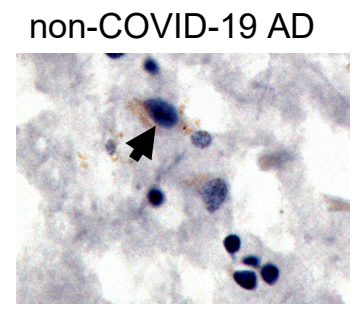

C

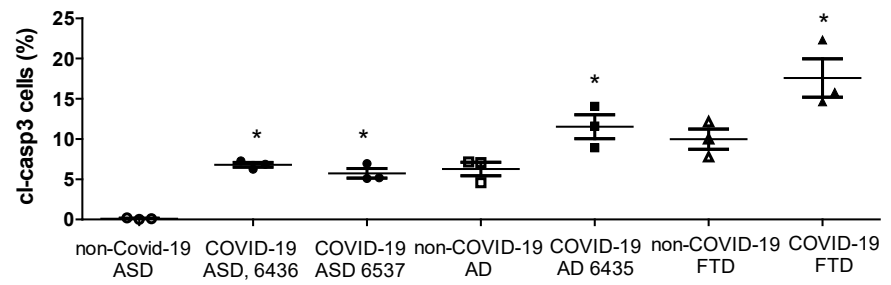

COVID-19 ASD 6436 COVID-19 ASD 6437 COVID-19 5932
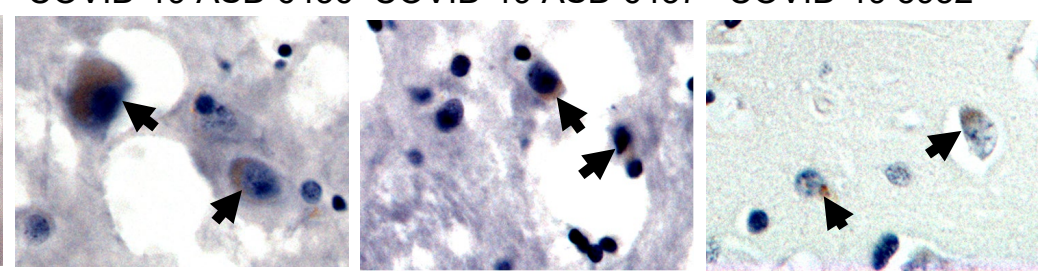

COVID-19 AD 6435

non-COVID-19 FTD

COVID-19 FTD 5922
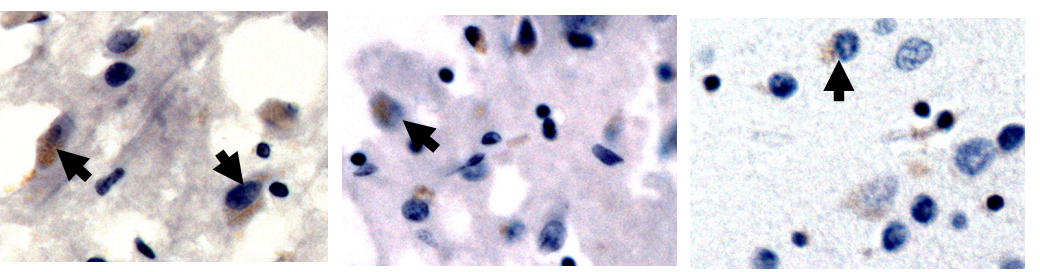
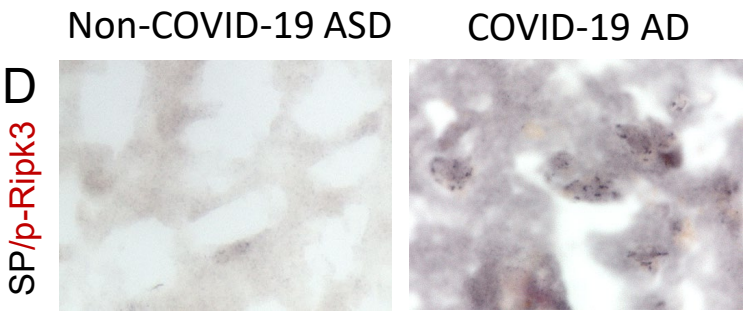

\section{COVID-19 ASD}

E
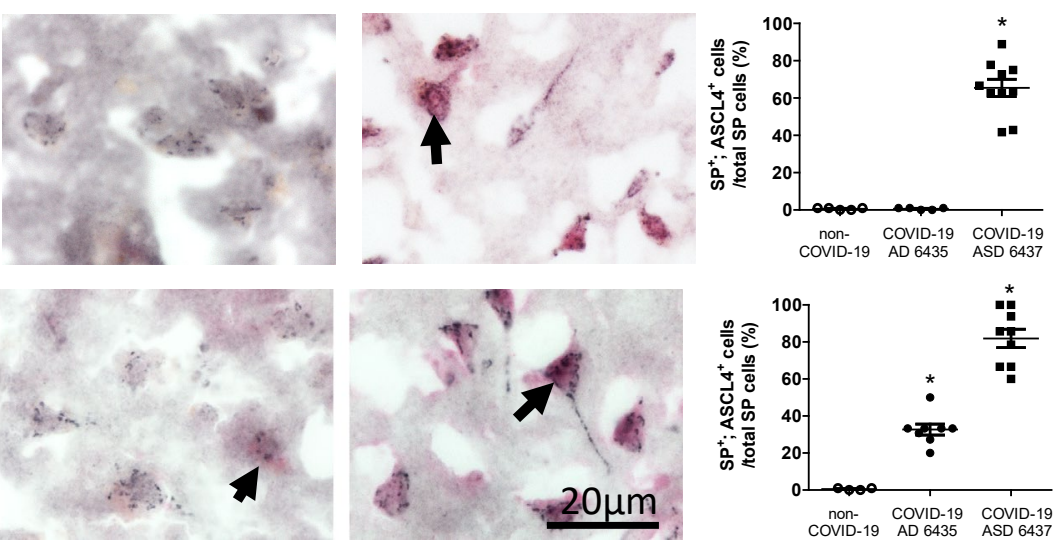
Fig. S4

A

non-COVID-19 ASD

COVID-19 ASD
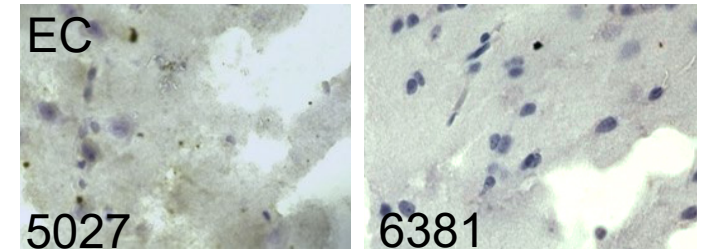

FC.

6381

6436

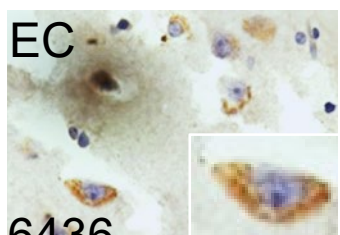

FC,

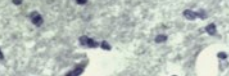

5027

B

EC

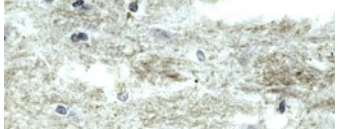

$5027 \quad 6381$
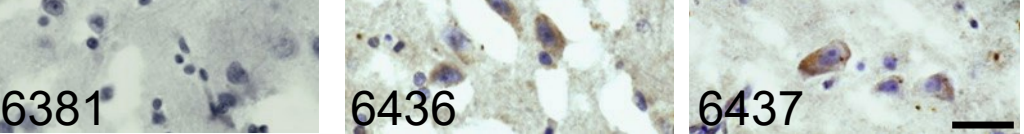

FC
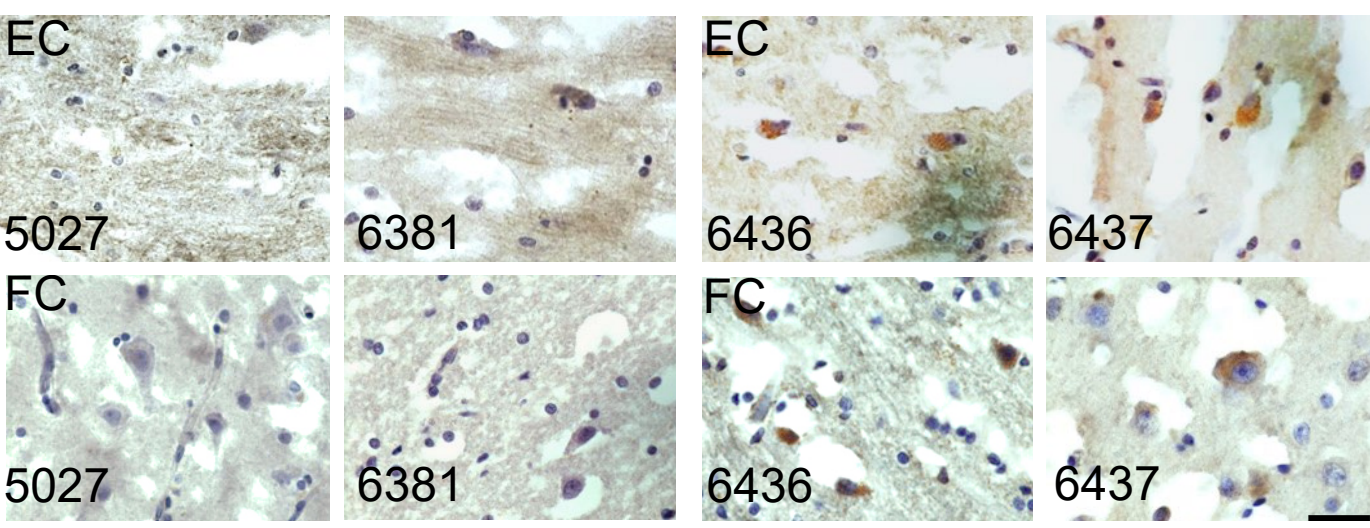

$6436-8=6437$

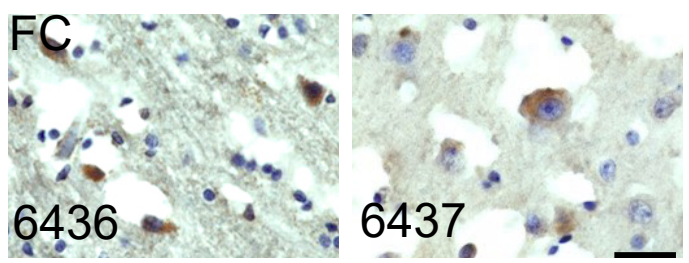

C

- Non-COVID-19 ASD

- COVID-19 ASD
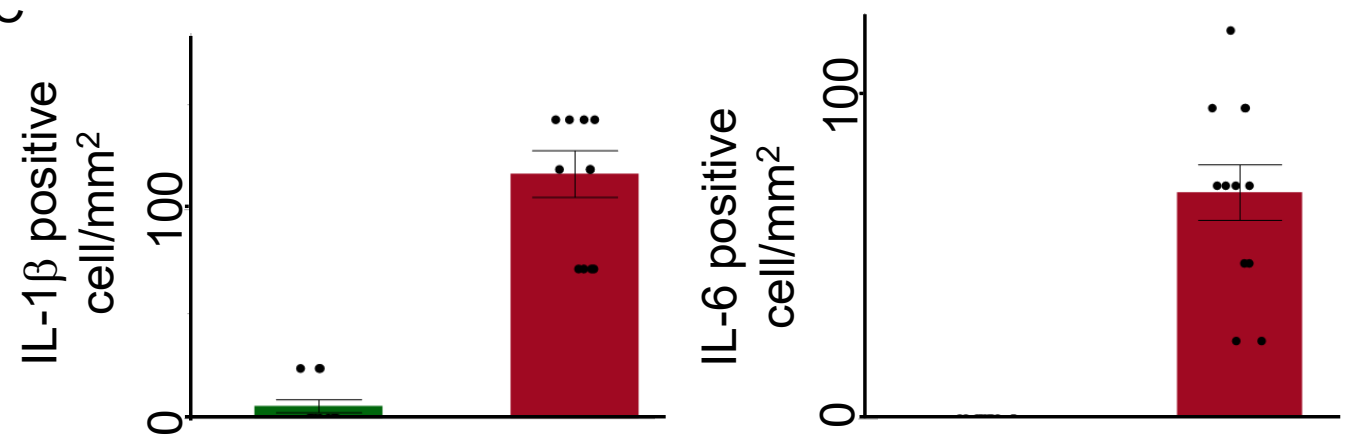
Fig. S5
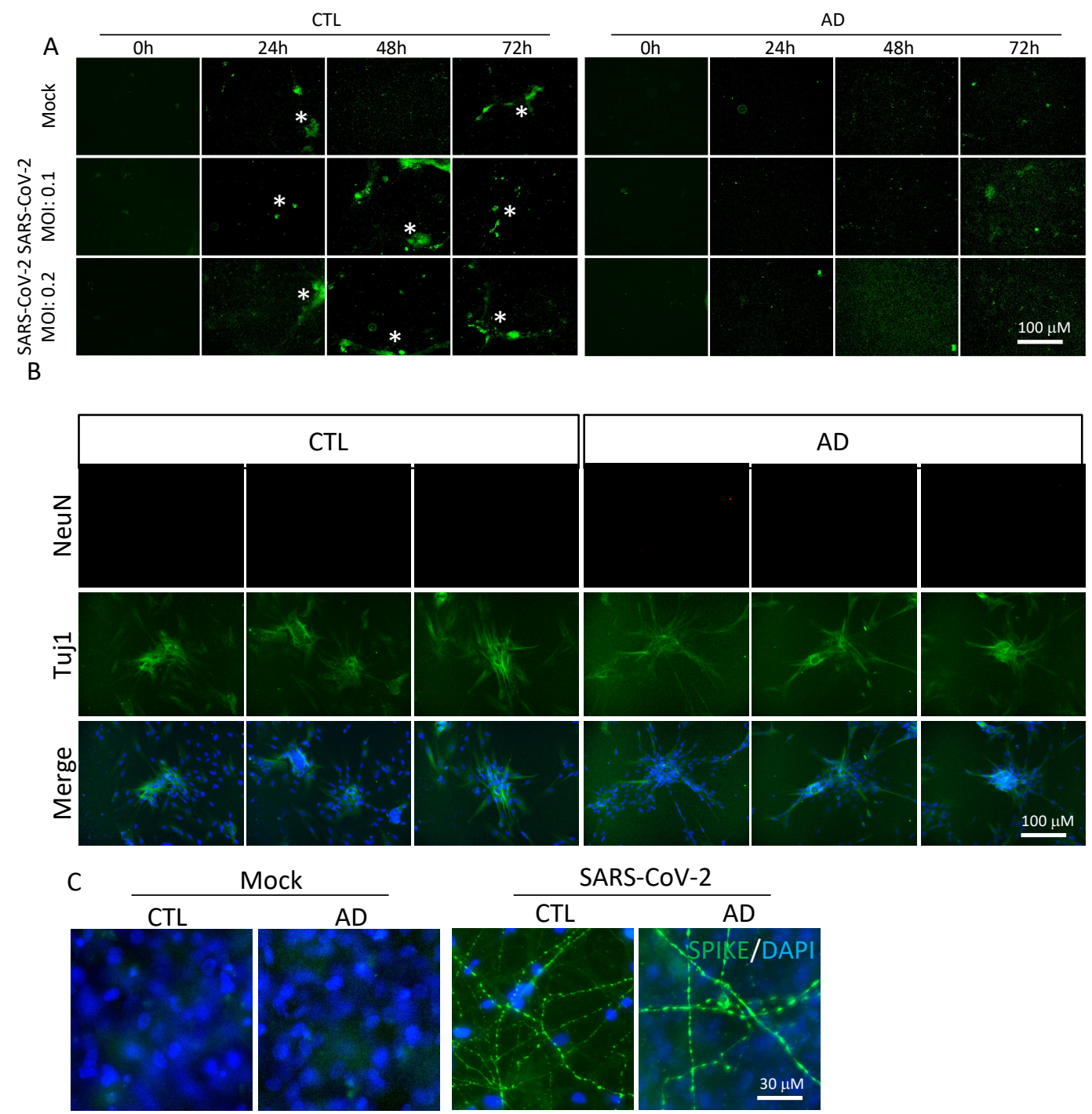

D

Mock

MOI:0.05

MOI:0.1

MOI:0.2

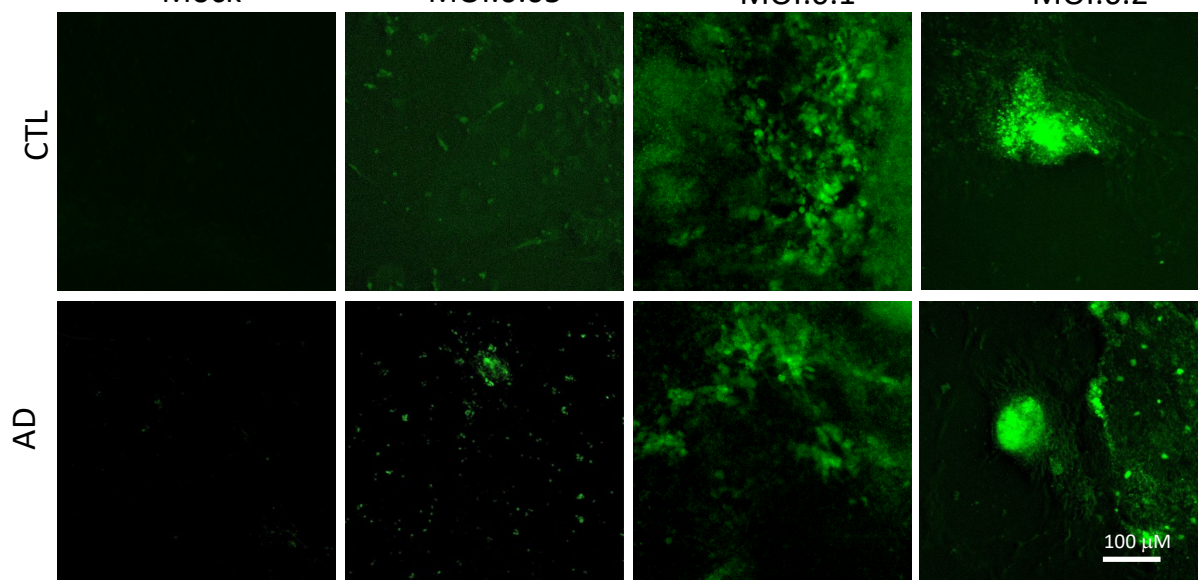


A

\begin{tabular}{|l|c|c|}
\hline Gene & CTL/AD & CTL/AD_V \\
\hline XAF1 & 1.60 & 3.58 \\
\hline TWIST2 & 1.81 & 3.96 \\
\hline CASP4 & 1.55 & 3.17 \\
\hline CD34 & 1.82 & 3.54 \\
\hline CCL4 & 2.83 & 5.49 \\
\hline CCL3 & 3.40 & 6.44 \\
\hline KLHDC7B & 1.79 & 3.33 \\
\hline LILRB1 & 4.37 & 8.12 \\
\hline GJB2 & 1.84 & 3.40 \\
\hline LAIR1 & 1.92 & 3.38 \\
\hline PLA2G7 & 2.08 & 3.66 \\
\hline C3 & 2.31 & 4.05 \\
\hline FIBIN & 1.67 & 2.85 \\
\hline HCK & 1.99 & 3.36 \\
\hline SP140L & 1.60 & 2.68 \\
\hline COMP & 1.69 & 2.83 \\
\hline SLC22A4 & 1.53 & 2.53 \\
\hline LRRC25 & 2.42 & 3.91 \\
\hline CTSS & 2.38 & 3.78 \\
\hline SLAMF8 & 2.16 & 3.36 \\
\hline FERMT3 & 3.28 & 5.09 \\
\hline NPL & 1.55 & 2.38 \\
\hline ITGAL & 2.83 & 4.32 \\
\hline AOAH & 2.29 & 3.48 \\
\hline CCL3L1 & 5.34 & 8.10 \\
\hline DE & & \\
\hline
\end{tabular}

Deteriorated fold $>1.5$

Upregulated genes

D
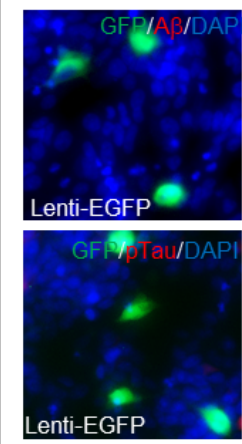

B

\begin{tabular}{|l|c|c|}
\hline Gene & CTL/AD & CTL/AD_V \\
\hline PLA2G2F & -1.58 & -5.46 \\
\hline CRYAA2 & -1.88 & -5.82 \\
\hline ABCB11 & -1.65 & -4.64 \\
\hline GJA8 & -1.81 & -5.06 \\
\hline CRYBB3 & -1.76 & -4.11 \\
\hline CRYBB1 & -2.58 & -5.90 \\
\hline C1orf105 & -1.65 & -3.70 \\
\hline CRYBA1 & -2.68 & -6.02 \\
\hline MIP & -3.11 & -6.95 \\
\hline CRYBA4 & -2.58 & -5.66 \\
\hline GPD1 & -1.60 & -3.43 \\
\hline BHMT & -2.52 & -5.31 \\
\hline PITX3 & -2.07 & -4.33 \\
\hline CRYBA2 & -3.24 & -6.72 \\
\hline GJA3 & -1.63 & -3.38 \\
\hline CRYGS & -2.73 & -5.65 \\
\hline PSG7 & -3.39 & -6.95 \\
\hline PSG3 & -3.17 & -6.45 \\
\hline KMO & -1.78 & -3.62 \\
\hline PSG2 & -3.57 & -7.24 \\
\hline CRYGD & -1.83 & -3.70 \\
\hline CRYAB & -2.33 & -4.53 \\
\hline CRYBB2 & -2.84 & -5.46 \\
\hline HSD3B1 & -1.60 & -2.96 \\
\hline PROX1 & -1.67 & -2.99 \\
\hline VIT & -1.68 & -2.99 \\
\hline ERICH5 & -1.59 & -2.80 \\
\hline AQP5 & -1.70 & -2.96 \\
\hline PSG1 & -2.66 & -4.61 \\
\hline COLGALT2 & -1.56 & -2.61 \\
\hline CRYGC & -2.69 & -4.45 \\
\hline ANKRD34C & -2.56 & -4.18 \\
\hline DBX2 & -2.75 & -4.29 \\
\hline MAMDC2 & -1.68 & -2.54 \\
\hline & & \\
\hline
\end{tabular}

C

$$
\text { Immune system }
$$
Signal transduction

Transport and catabolism

Signaling molecules and

Cancers: Overview

Infectious diseases: Viral

Infectious diseases: Bacterial

Infectious diseases: Parasitic

Immune diseases

Development

Endocrine and metabolic diseases

Endocrine system

Cellular community - eukaryotes

Cardiovascular diseases

Cancers: Specific types

Cell motility

Amino acid metabolism

Drug resistance: Antineoplastic

Neurodegenerative diseases

Cell growth and death

Sensory system

Circulatory system

Nervous system

Environmental adaptation

Excretory system Aging

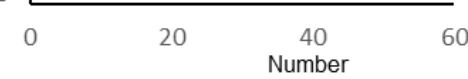

KEGG Pathway Classification

Signal transduction Amino acid metabolism Endocrine system

Signaling molecules and interaction Endocrine and metabolic diseases

Folding, sorting and degradation

Environmental adaptation

Aging

Drug resistance: Antineoplastic

Membrane transport

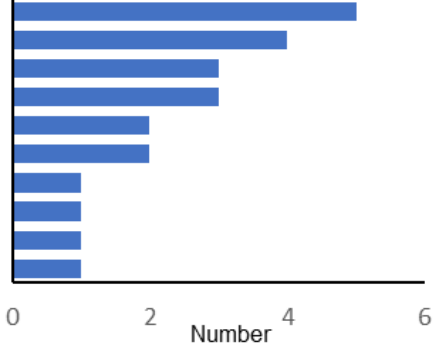

E
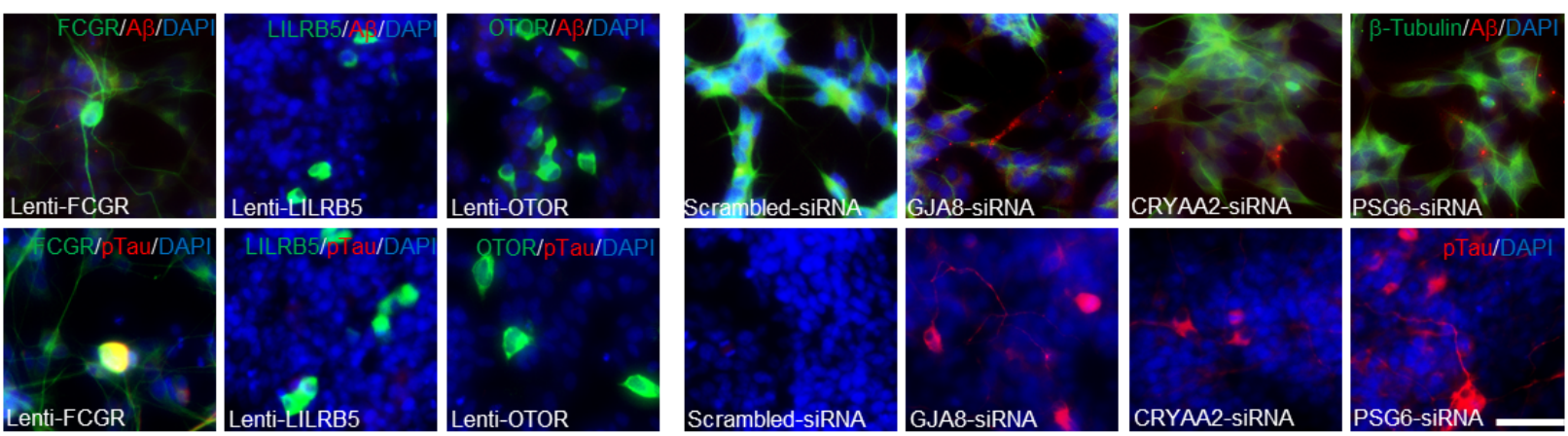


\section{Resource Tables}

\section{Antibody}

Rabbit anti-SARS-CoV-2 spike glycoprotein antibody

Rabbit anti-SARS Nucleocapsid Protein Antibody

Rabbit anti-human ACE2 polyclonal antibody

rabbit anti-neuropilin-1 antibody

Mouse anti-Glutamate Decarboxylase, 65 kDa Isoform (GAD65), clone GAD-6 antibody

Mouse anti-Glutamine Synthetase, clone GS-6 antibody

Rabbit anti-NeuN antibody

Anti-Galactocerebroside antibody produced in rabbit

Rabbit anti-Glial Fibrillary Acidic Protein (GFAP) antibody

Anti- $\beta$-Amyloid, 1-16 Antibody (Previously Covance catalog\# Biolegend SIG-39300)

$\beta$-Amyloid (D3D2N) Mouse mAb

Rabbit anti-phospho-Tau (Ser202, Thr205) Monoclonal Antibody (AT8)

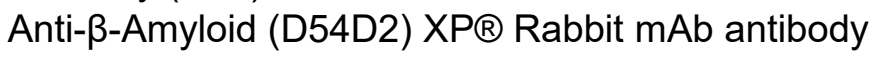

Neurofilament-L (C28E10) Rabbit mAb antibody

Rabbit anti-Cleaved Caspase-3 (Asp175) Antibody

Neuronal Class III beta-Tubulin (TUJ1) Mouse Monoclonal Antibody, Purified

Rabbit anti Iba1, Rabbit antibody

Rabbit anti-RIP3 (phospho S227) antibody [EPR9627]

Rabbit anti-MLKL (phospho S358) antibody

Mouse anti-TRANSFERRIN RECEPTOR Monoclonal Antibody (H68.4)

Covance
Sources

Abcam

Novus

Thermo Fisher

Scientific

Abcam

millipore

millipore

Abcam

Sigma-Aldrich

Agilent

Cell Signaling

Technology

Thermo Fisher

Scientific

Cell Signaling

Technology

Cell Signaling

Technology

Cell Signaling

Technology

FUJIFILM Wako

Abcam

Abcam

Thermo Fisher Scientific

\section{Identifier}

Cat\# ab272504; RRID:

AB_2847845

Cat\# NB100-56576;

RRID:AB_838838

Cat\# PA5-20046;

RRID: AB_1115262

Cat\# ab81321; RRID:

AB_164073

Cat\# mAB351; RRID:

AB_2263126

Cat\# MAB302; RRID:

AB_2110656

Cat\# ab177487; RRID:

AB_2532109

Cat\# 9152; RRID:

AB_259984

Cat\# Z0334; RRID:

AB_10013382

Cat\# 803014;

RRID:AB_2728527

Cat\# 15126;

RRID:AB_2798720

Cat\# MN1020; RRID:

AB_223647

Cat\# 8243; RRID:

AB 2797642

Cat\# 2837; RRID:

AB 823575

Cat\# 9661; RRID:

AB_2341188

Cat\# MMS-435P;

RRID: AB 231377

Cat\# 019-19741;

RRID:AB 839504

Cat\# ab209384; RRID:

AB_2714035

Cat\# ab187091; RRID: AB_2619685

Cat\# 13-6800; RRID: AB_86623 
bioRxiv preprint doi: https://doi.org/10.1101/2022.01.31.478476; this version posted February 3, 2022. The copyright holder for this preprint (which was not certified by peer review) is the author/funder. All rights reserved. No reuse allowed without permission.

Rabbit anti-FACL4 (ASCL4) antibody [EPR8640]

Rabbit anti-DPP4/CD26 (D6D8K) antibody

Rabbit anti-phospho-Tau (Ser396) Polyclonal Antibody

Rabbit anti-phospho-Tau (Ser214) Polyclonal Antibody

Rabbit anti-pTau-Ser202(PS202) antibody

Rabbit Anti-Amyloid oligomer, abeta, oligomeric antibody

Human IL-1 beta/IL-1F2 MAb (Clone 8516) antibody

Mouse Anti-Human IL-6 Monoclonal antibody, Unconjugated, R and D System Clone 1936

CD3 Monoclonal Antibody (OKT3), Functional Grade

Mouse anti-human CD20 antibody

F8/40 mouse monoclonal antibody

Biotin-conjugated Goat anti-mouse IgG

Biotin-conjugated Goat anti-Rabbit IgG

Alexa488-donkey anti-mouse lgG

Alexa488-donkey anti-rabbit lgG

Alexa594-donkey anti-mouse IgG

Alexa594-doneky anti-rabbit lgG

Goat anti-mouse lgG, alkaline phosphatase conjugated

Goat anti-rabbbit IgG, alkaline phosphatase conjugated

Histofine Simple Stain MAX PO (R)

Histofine Simple Stain MAX PO (M)
Abcam

Cell Signaling

Tech

Thermo Fisher

Scientific

Thermo Fisher

Scientific

In house

Millipore

$R$ and D System

Thermo Fisher

Scientific

Biolegend

Santa Cru Biotech

Vector Lab

Vector Lab

Invitrogen

Invitrogen

Invitrogen

Invitrogen

thermo Fisher

Scientific

thermo Fisher

Scientific

Nichirei Bioscience

Nichirei Bioscience Cat\# 414131F
Cat\# ab155282; RRID:

AB_2714020

Cat\# 67138; RRID:

AB_2728750

Cat\# 44-752G; RRID:

AB 253374

Cat\# 44-742G; RRID:

AB_2533740

Cat\# AB9234; RRID:

AB_570955

Cat\# MAB201;

RRID:AB_358006

Cat\# MAB2061;

RRID: AB_2127616

Cat\# 16-0037-81;

RRID: AB_468854

Cat\# 302301;

RRID:AB_314249

Cat\# sc377009

Cat\# BA-9200;

RRID:AB_2336171

Cat\# BA-1000;

RRID:AB 2313606

Cat\# A21201;

RRID:AB_141630

Cat\# A21206;

RRID:AB_2535792

Cat\# A21203;

RRID:AB_141633

Cat\# A21207;

RRID:AB_141637

Cat\# A21060;

RRID:AB 2536528

Cat\# A16099; RRID:

AB_2534773

Cat\# 414141F 
bioRxiv preprint doi: https://doi.org/10.1101/2022.01.31.478476; this version posted February 3, 2022. The copyright holder for this preprint (which was not certified by peer review) is the author/funder. All rights reserved. No reuse allowed without permission.

Viral strain

SARS-CoV-2, WA-1 strain - BEI

SARS-CoV-2 GFP

CMV Promoter Thomson Factors Lentivirus Set
Sources

CDC

provided by Dr. Ralph S. Baric

ABMgood, Canada
Identifier

Cat \#NR-52281

Hou et al.

Cat\# G353 
Reagent or Resources

Normal Donkey Serum

PBS, $\mathrm{pH} 7.4$

Triton X100

Hydrogen peroxide ( $\mathrm{H} 2 \mathrm{O} 2)$

Antigen Retriever, Citrate buffer, pH6

Antigen Retriever, formic acid

VECTASTAIN Elite Kits

SignalStain $\AA$ Vibrant Red AP Substrate Kit

DAB (3,3'-Diaminobenzidine tetrahydrochloride hydrate)

Hematoxylin Solution, Harris Modified

Tissue-Plus OCT Compound

Ammonium Nickel (II) sulfate hexahydrate

Lithium Carbonate

Ethanol, Decon Labs, 200 proof

Xylenes Histological Grade

Thioflavin-T

DAPI (4',6-diamidino-2-phenylindole)

Permount mounting medium

Fluorescence mounting medium

Paraformaldehyde (4\%, pH7.4) in PBS

RNAscope $\circledast 2.5$ HD Reagent Kit-RED

RNAscope ${ }^{\circledR}$ Probe

Knockout DMEM medium

Knockout serum replacer

Nonessential amino acids

GlutaMAX

$\beta$-mercaptoethanol

Recombinant human basic fibroblast growth factor

Collagenase IV

DMEM/F12

N2 supplement

Neurobasal medium
Sources

Sigma

Quality Biological

Sigma

Sigma

Sigma

Sigma

VectorLab

Cell Signal Tech

Sigma

Sigma

Fisher Scientific

Sigma

Sigma

VWR

Sigma

Sigma

Thermo Fisher

Scientific

Fisher

Sigma

Thermo Fisher

Scientific

ACD

$A C D$

Thermo Fisher

Scientific

Thermo Fisher

Scientific

Thermo Fisher

Scientific

Thermo Fisher

Scientific

Sigma

Thermo Fisher

Scientific

Stem Cell

Technologies

Thermo Fisher

Scientific

Thermo Fisher

Scientific

Thermo Fisher

Scientific
Identifier

Cat\# D9663

Cat\# 119-069-131

Cat\# X100

Cat\# H1009

Cat\# C9999

Cat\# F0507

Cat\# PK-6100

Cat\# 76713

Cat\# D5637

Cat\# HHS32

Cat\# 23-730-571

Cat\# A1827

Cat\# 255823

Cat\# 71002-426

Ca\# 534056

Cat\# T3516

Cat\# D9542

Cat\# SP15100

Cat\# F4680

Cat\# J61889

Cat\# 322350

Cat\# 854841

Cat\# 10829018

Cat\# 10828028

Cat\# 11140050

Cat\# 35050061

Cat\# M3148

Cat\# 13256-029

Cat\# 17104019

Cat\# 11320033

Cat\# 17502048

Cat\# 21103049 
B27 supplement

Penicillin/streptomycin

Stemolecule SB431542, TGF- $\beta$ inhibitor

LDN-193189, inhibitor of bone morphogenetic protein receptor

Matrigel

bFGF

EGF, epidermal growth factor

Heparin

Brain-derived neurotrophic factor, BDNF

Glial cell-derived neurotrophic factor, GDNF

X-tremeGENE siRNA transfection reagent, Roche

SuperScript ${ }^{\mathrm{TM}}$ III Reverse Transcriptase kit

PowerUp $^{\text {TM }}$ SYBR ${ }^{\text {TM }}$ Green Master Mix
Thermo Fisher

Scientific

Cat 17504044

Thermo Fisher

Scientific

Reprocell

Reprocell

BD Biosciences

Pepro Tech

Pepro Tech

Sigma

Pepro Tech

Pepro Tech

Sigma

Cat\# 15140122

Cat\# 04-0010-10

Cat\# 04-0074

Cat\# 354277

Cat\# 100-18b

Cat\# 100-47

Cat\# H4784

Cat\# 450-02

Cat\# 450-10

Cat\# 6366236001

Thermo Fisher

Scientific

Thermo Fisher

Scientific
Cat\# 18080051

Cat\# A25742 
Brain Tissue

Source

Alzheimer's Disease (AD)-Covid19 brain tissue, Male, NIH NeuroBioBank Autism-Covid19 brain tissue, Female, $38 \mathrm{yr}$

Autism-Covid19 brain tissue, Male, $30 \mathrm{yr}$

Frontotemporal Dementia (FTD)-Covid19, Female, 71 UCSD, $\mathrm{yr}$

Covid19, Male, $77 \mathrm{yr}$

Sporadic AD, Female, $65 \mathrm{yr}$

Sporadic AD, Male, $63 \mathrm{yr}$

Sporadic AD, Male, $63 \mathrm{yr}$

Sporadic AD, Male, $79 \mathrm{yr}$

Sporadic AD, Female, $71 \mathrm{yr}$

Sporadic AD, Male, $76 \mathrm{yr}$

Unaffected Control, Male, 29 yr

Unaffected Control, Male, $30 \mathrm{yr}$

Unaffected Control, Female, $38 \mathrm{yr}$

Unaffected Control, Male, $38 \mathrm{yr}$

Unaffected Control, Male, 38 yr

Unaffected Control, Female, 77 yr

Unaffected Control, Male, 75 yr

Unaffected Control, female, 76 yr

ASD-Autism, $37 \mathrm{yr}$

ASD-Autism suspended, Epilepsy, 28 yr

ASD-Autism, Epilepsy, $29 \mathrm{yr}$

ASD-Austim suspended, $35 \mathrm{yr}$

ASD-Autism suspended, $36 \mathrm{yr}$

ASD-Autism suspended, $30 \mathrm{yr}$

Frontotemporal dementia, $70 \mathrm{yr}$

Frontotemporal dementia, 65yr

Frontotemporal dementia, 76yr

Frontotemporal dementia, 73yr
NIH NeuroBioBank

NIH NeuroBioBank

UCSD,

NIH NeuroBioBank

NIH NeuroBioBank

NIH NeuroBioBank

UCSD,

UCSD,

UCSD,

NIH NeuroBioBank

NIH NeuroBioBank

NIH NeuroBioBank

NIH NeuroBioBank

NIH NeuroBioBank

NIH NeuroBioBank

NIH NeuroBioBank

NIH NeuroBioBank

NIH NeuroBioBank

NIH NeuroBioBank

NIH NeuroBioBank

NIH NeuroBioBank

NIH NeuroBioBank

NIH NeuroBioBank

NIH NeuroBioBank

NIH NeuroBioBank

NIH NeuroBioBank

NIH NeuroBioBank

\section{Identifier}

UMBN \#6435

UMBN \#6436

UMBN \#6437

\#5922

\#5932

\#116311

\#116319

\#116317

\#5877

\#5875

\#5872

UMBN \#1502

UMBN \#6099

UMBN \#1648

UMBN \#5249

UMBN \#4592

UMBN \#1569

UMBN \#5830

UMBN \#5219

UMBN \#5027

UMBN \#5934

UMBN \#5940

UMBN \#6092

UMBN \#6212

UMBN \#6381

UMBN \#6220

UMBN \#6019

UMBN \#6264

UMBN \#6207 


\section{Cell lines}

iPSCs, from Familial AD, female, $31 \mathrm{yr}$ iPSCs, from Sporadic AD, male, $60 \mathrm{yr}$ iPSCs, from Sporadic AD, male, $83 \mathrm{yr}$ iPSCs, from apparently healthy male, $55 \mathrm{yr}$ iPSCs, from apparently healthy female, $36 \mathrm{yr}$ iPSCs, from apparently healthy female, $36 \mathrm{yr}$ Primary human neuron

Vero E6 cells

mouse embryonic fibroblasts (MEF)

\section{Source}

Coriell Institute Coriell Institute Coriell Institute Coriell Institute Coriell Institute Coriell Institute Neuromics

ATCC Thermo Fisher Scientific

\section{Identifier}

Cat\# AG25367

Cat\# AG07376

Cat\# GM24666

Cat\# GM23248

Cat\# GM23279

Cat\# GM23280

HNC001

CRL-1586

Cat\# A34180 


\section{Plasmid DNAs}

CRYAA2_pcDNA3.1+/C-(K)-DYK

GJA8_pcDNA3.1+/C-(K)-DYK

OTOR(NM_020157.4) ORF Clone

Human LILRB5(NM_001081442.2) ORF Clone

FCGR3A(NM_000569.6) ORF Clone

PSG6(NM_001031850.4) ORF Clone

\section{SiRNAs}

GJA8 SiRNA

CRYAA2 SIRNA

PSG6 SiRNA

\section{Lentiviru}

Otoraplin (OTOR) (NM_020157) Human Tagged ORF Clone

LILRB5 (NM_001081443) Human Tagged ORF

CD16 (FCGR3A) (NM_000569) Human Tagged ORF

$\begin{array}{ll}\begin{array}{ll}\text { Source } \\ \text { Genscript } \\ \text { Genscript } \\ \text { Genscript } \\ \text { Genscript } \\ \text { Genscript } \\ \text { Genscript }\end{array} & \begin{array}{l}\text { Identifier } \\ \text { Cat\# OHu53183D } \\ \text { Cat\# OHu22749D } \\ \text { Cat\# OHu00520D } \\ \text { Cat\# OHu64562D } \\ \text { Cat\# OHu27381D } \\ \text { Cat\# OHu56074D }\end{array} \\ \begin{array}{l}\text { Source } \\ \text { Ambion }\end{array} & \begin{array}{l}\text { Identifier } \\ \text { Ambion }\end{array} \\ \text { Ambion } & \begin{array}{l}\text { Cat\# 354769 } \\ \text { Cat\# 11312 }\end{array} \\ \text { Source } & \text { Identifier } \\ \text { Origene } & \text { Cat\# RC210984L4V } \\ \text { Origene } & \text { Cat\# RC219303L4V } \\ \text { Origene } & \text { Cat\# RC206429L2V }\end{array}$


bioRxiv preprint doi: https://doi.org/10.1101/2022.01.31.478476; this version posted February 3, 2022. The copyright holder for this preprint (which was not certified by peer review) is the author/funder. All rights reserved. No reuse allowed without permission.

Software and Algorithems

GraphPad Prism 7

photoshop CS3
Source

GraphPad

Adobe $\underline{\text { Identifier }}$

NA

NA 
bioRxiv preprint doi: https://doi.org/10.1101/2022.01.31.478476; this version posted February 3, 2022. The copyright holder for this preprint (which was not certified by peer review) is the author/funder. All rights reserved. No reuse allowed without permission.

\section{QPCR primers}

FCGR3A qPCR, mRNA forward: 5'-CCTCCTGTCTAGTCGGTTTGG; reverse: 5'- Genbank Accession TCGAGCACCCTGTACCATTGA

LILRA3 qPCR, mRNA forward: 5'-AGGAGTGGGGACGTGACTT; reverse: 5'GGTCTGGCACGGATCTGTC

TLR8 qPCR, mRNA forward: 5'-AACTGCCAAGCTCCCTACG; reverse: 5'CAAGGCACGCATGGAAATGG

LILRB5 qPCR, mRNA forward: 5'-GACTGATCCCTGACATACCCG; reverse: 5'GTGTCTATCTGATGCCATGACTG

OTOR qPCR, mRNA forward: 5'-TCTGGCTAGTGCTCAAGAAGA; reverse: 5'TAACCCACGACTCCCATCTCG

TMEM140 qPCR, mRNA forward: 5'-TCGGCTTCTATAACTTCTGCCT; reverse: 5'-CTGTTGCACTGGGCTAGGAG

SIGLEC1 qPCR, mRNA forward: 5'-ATGGGGTACGCCTCCAAAC; reverse: 5'GTGCCTCATTGGGTGTGTTG

CCL4 qPCR, mRNA forward: 5'-CTGTGCTGATCCCAGTGAATC; reverse: 5'TCAGTTCAGTTCCAGGTCATACA

SLCO2B1 qPCR, mRNA forward: 5'-TATGTGGACATTAACCAGATGCC; reverse: 5'-CTGTGACTGCTAAGACCTTTCG

TM4SF4 qPCR, mRNA forward: 5'-CTGTGGTTGGATTCTTGGGAG; reverse: 5'-GGGTAGCCCCATGTACTATTGG

XAF1 qPCR, mRNA forward: 5'-GCTCCACGAGTCCTACTGTG; reverse: 5'-

GTTCACTGCGACAGACATCTC

KCNQ5 qPCR, mRNA forward: 5'-TGTTGTCGATATAGAGGATGGCA;

reverse: 5'-GAGTGCAGACGTGGCAAAAAT

ANGPTL1 qPCR, mRNA forward: 5'-AGAAAGGAAAGCCGTAACATGAA; reverse: 5'-TCCCTGTATCTTGTTGCCATCT

COL21A1 qPCR, mRNA forward: 5'-GGCCACAAATAGCAGTTACCT; reverse: 5'-AGCCTTCATCAAACAACGTCTTA

KLHDC7B qPCR, mRNA forward: 5'-GCACCATGCACAACTACCTGT; reverse: 5'-ATTCGCCACCGATGGCATAG

HAND2 qPCR, mRNA forward: 5'-CGCCGACACCAAACTCTCC; reverse: 5'TCGCCATTCTGGTCGTCCT

COMP qPCR, mRNA forward: 5'-CGAGTCCGCTGTATCAACACC; reverse: 5'TCCGTGCAAACCTGCTTGT

SLC22A4 qPCR, mRNA forward: 5'-TGGTAGCCTTCATACTAGGAACA; reverse: 5'-TGGCAGCAGCATATAGCCAAC

PTPRH qPCR, mRNA forward: 5'-TCGCCCTATGCTGGGAAGT; reverse: 5'TCTGCCCATACAGAGAATGTGT

FGF10 qPCR, mRNA forward: 5'-CATGTGCGGAGCTACAATCAC; reverse: 5'CAGGATGCTGTACGGGCAG

VIT qPCR, mRNA forward: 5'-TGTGCTTGATAATTCAGGAGGGA; reverse: 5'GATTGGACACCGTTGGAATAACT

GJA8 qPCR, mRNA forward: 5'-GACCCTGCTGAGGACCTACAT; reverse: 5'CCCAACTCCATCACGTTGAG

\section{Sources}

NM_000569

Genbank Accession

NM_006865

Genbank Accession

NM_138636

Genbank Accession

NM_006840

Genbank Accession

NM_020157

Genbank Accession

NM_018295

Genbank Accession

NM_023068

Genbank Accession

NM_002984

Genbank Accession

NM_001145211

Genbank Accession

NM_004617

Genbank Accession

NM_017523

Genbank Accession

NM_001160134

Genbank Accession

NM_004673

Genbank Accession

NM_030820

Genbank Accession

NM_138433

Genbank Accession

NM_021973

Genbank Accession

NM_000095

Genbank Accession

NM_003059

Genbank Accession

NM_002842

Genbank Accession

NM 004465

Genbank Accession NM_001177970

Genbank Accession NM_005267
Identifier

PrimerBank ID 24429586a1

PrimerBank ID 289547630c1

PrimerBank ID 257196253c3

PrimerBank ID 125987589c2

PrimerBank ID 21618345c1

PrimerBank ID 256355110c2

PrimerBank ID 89142743c1

PrimerBank ID 4506845a1

PrimerBank ID 312176373c2

PrimerBank ID 325974483c2

PrimerBank ID 378925606c1

PrimerBank ID 236462241c3

PrimerBank ID 38327520c3 PrimerBank ID 90577174c2 PrimerBank ID 206597555c1 PrimerBank ID 88999597c2 PrimerBank ID 40217842c2

PrimerBank ID 24497489c1

PrimerBank ID 241896923c2

PrimerBank ID 4758359c1

PrimerBank ID 295789106c2 PrimerBank ID 281182631c2 
bioRxiv preprint doi: https://doi.org/10.1101/2022.01.31.478476; this version posted February 3, 2022. The copyright holder for this preprint (which was not certified by peer review) is the author/funder. All rights reserved. No reuse allowed without permission.

PSG6 qPCR, mRNA forward: 5'-AAGCTGCCCATGCCTTACATC; reverse: 5'- Genbank Accession PrimerBank ID AGGTGTAGTTCCGACTCTTAGG

NM_001031850

$141802170 c 1$

Amyloid precursor protein (APP), mRNA, forward: 5'-

GTCTCTCTCCCTGCTCTACAA; reverse: 5'-GGCCAAGACGTCATCTGAATAG

Genbank Accession Cairns et al.,

NM_000484 $\underline{2020}$

ABeta secretase 1 (BACE1), mRNA, forward: 5'-CCATCCTTCCGCAGCAATA; reverse: 5'-CGTAGAAGCCCTCCATGATAAC

Genbank Accession

NM_012104

Cairns et al.,

Tumor necrosis factor alpha (TNFa), mRNA, forward: 5'-

GAGGCCAAGCCCTGGTATG; reverse: 5'-CGGGCCGATTGATCTCAGC

Genbank Accession

NM_000594.4

$\underline{2020}$

Cairns et al.,

$\underline{2021}$

Presenilin 1 (PSEN1), mRNA, forward: 5'-TGGCTACCATTAAGTCAGTCAGC; reverse: 5'-CCCACAGTCTCGGTATCTTCT

Genbank Accession

NM_000021

Cairns et al.,

$\underline{2024}$

Presenilin 2 (PSEN2), mRNA, forward: 5'-CTGACCGCTATGTCTGTAGTGG;

reverse: 5'-CTTCGCTCCGTATTTGAGGGT

Genbank Accession

NM_012486

Cairns et al., $\underline{2025}$

Interleukin 6 (IL-6), mRNA, forward: 5'-TCAATATTAGAGTCTCAACCCCC;

reverse: 5'-TTGTTTTCTGCCAGTGCCTC

Genbank Accession

NM_012486

Cairns et al., $\underline{2026}$

Interleukin 1beta (IL-1 $\beta$ ), mRNA, forward: 5'-CAGAAGTACCTGAGCTCGCC; Genbank Accession reverse: 5'-AGATTCGTAGCTGGATGCCG

NM_000576.2

Cairns et al., $\underline{2027}$

Interferon gamma (INFY), mRNA, forward: 5'-ACTGTCGCCAGCAGCTAAAA; Genbank Accession reverse: 5'-TATTGCAGGCAGGACAACCA NM_000619 $\underline{2028}$

Glyceraldehyde 3-phosphate dehydrogenase (GAPDH), mRNA, forward: 5'- Genbank Accession ATTGCCCTCAACGACCACT; reverse: 5'-ATGAGGTCCACCACCCTGT NM_002046.5

Cairns et al., $\underline{2030}$

CDC 2019-nCoV_N1, forward: 5'-GAC CCC AAA ATC AGC GAA AT

IDT, Cat\#

CDC 2019-nCoV_N1, reverse: 5'-TCT GGT TAC TGC CAG TTG AAT CTG

$\underline{\mathrm{CDC}}$

10006821

IDT, Cat\#

10006822

2019-nCoV_N_Positive Control

$\underline{\text { IDT }}$

IDT, Cat\#

10006625 